\title{
Long-range inhibitory intersection of a retrosplenial thalamocortical circuit by apical tuft-targeting CA1 neurons
}

\author{
Naoki Yamawaki ${ }^{1}$, Xiaojian Li $^{1}$, Laurie Lambot ${ }^{1}$, Lynn Y. Ren ${ }^{2}$, Jelena Radulovic ${ }^{1,2}$, and \\ Gordon M. G. Shepherd ${ }^{1}$ \\ ${ }^{1}$ Department of Physiology, Feinberg School of Medicine, Northwestern University, Chicago, \\ Illinois 60611; \\ ${ }^{2}$ Department of Psychiatry and Behavioral Sciences, Feinberg School of Medicine, Northwestern \\ University, Chicago, Illinois 60611
}

\section{Abstract}

\begin{abstract}
Hippocampus, granular retrosplenial cortex (RSCg), and anterior thalamic nuclei (ATN) interact to mediate diverse cognitive functions. To identify cellular mechanisms underlying hippocampothalamo-retrosplenial interactions, we investigated the potential circuit suggested by projections to RSCg layer 1 (L1) from GABAergic CA1 neurons and ATN. We find that CA1 $\rightarrow$ RSCg projections stem from GABAergic neurons with a distinct morphology, electrophysiology, and molecular profile. Their long-range axons inhibit L5 pyramidal neurons in RSCg via potent synapses onto apical tuft dendrites in L1. These inhibitory inputs intercept L1-targeting thalamocortical excitatory inputs from ATN to co-regulate RSCg activity. Subicular axons, in contrast, excite proximal dendrites in deeper layers. Short-term plasticity differs at each connection. Chemogenetically abrogating $\mathrm{CA} 1 \rightarrow \mathrm{RSCg}$ or ATN $\rightarrow \mathrm{RSCg}$ connections oppositely affects the encoding of contextual fear memory. Our findings establish retrosplenial-projecting CA1 neurons as a distinct class of long-range dendrite-targeting GABAergic neuron, and delineate an unusual cortical circuit specialized for integrating long-range inhibition and thalamocortical excitation.
\end{abstract}

\section{Introduction}

GABAergic projection neurons - inhibitory neurons with long-range axons - are important circuit elements in many brain areas, such the corticonuclear projections of Purkinje cells in

\footnotetext{
Users may view, print, copy, and download text and data-mine the content in such documents, for the purposes of academic research, subject always to the full Conditions of use:http://www.nature.com/authors/editorial_policies/license.html\#terms

Correspondence: naoki.yamawaki@northwestern.edu or g-shepherd@northwestern.edu.

Author Contributions: N.Y., J.R., and G.S. designed research; N.Y. performed injections, ex vivo recording, imaging,

immunostaining, and reconstruction; X.L. performed in vivo recording; L.L performed immunostaining and reconstruction. L.R. and J.R. performed behavioral studies; N.Y., J.R, and G.S. analyzed data and wrote the paper.

Reporting Summary. Further information on research design is available in the Nature Research Reporting Summary linked to this article.

Code availability. Software routines for morphological analysis are available from ref. ${ }^{58}$ or the corresponding authors, and digital reconstructions will be available at NeuroMorpho.org.

Data availability statement: The data are available upon reasonable request.

Competing Financial Interests Statements: The authors declare no competing interests.
} 
the cerebellum and the striatonigral and striatopallidal projections of spiny projection neurons in the basal ganglia. In neocortical circuits, however, inhibition is mostly locally sourced, engaged through potent feedforward excitation of interneurons by excitatory afferents ${ }^{1}$. Several exceptions have been identified, including inhibitory afferents to neocortex from somatostatin-expressing neurons in the zona incerta that project to sensorimotor corte ${ }^{2,3}$, GABAergic neurons in the globus pallidus that project to frontal cortex $^{4}$, somatostatin-expressing neurons within the neocortex that project corticocortically to ipsilateral areas ${ }^{5,6}$, and parvalbumin-expressing neurons that project via corpus callosum to contralateral cortex 7,8 . For several of these, progress has been made toward characterizing both the presynaptic neurons and the long-range inhibitory circuits they form with postsynaptic cortical neurons.

A GABAergic projection from hippocampus to parietal cortex has also been described anatomically, arising from neurons in dorsal CA1 at the stratum radiatum/lacunosummoleculare border that send axons to the granular retrosplenial cortex (RSCg), ramifying in $\mathrm{L} 1^{9}, 10$. Unlike the neocortically projecting GABAergic neurons mentioned above, however, the cellular properties of the presynaptic neurons are obscure, and the targets of their axons in the RSCg are unknown.

This GABAergic $\mathrm{CA} 1 \rightarrow \mathrm{RSCg}$ projection lies within a larger network thought to support the encoding and storage of hippocampally derived information in the $\mathrm{RSCg}$ (reviewed in refs. ${ }^{11-13}$ ). Understanding how the GABAergic neurons in CA1 are connected to postsynaptic RSCg neurons could thus yield mechanistic insight into the cellular basis for behaviors mediated by hippocampo-retrosplenial communication in this system.

Hippocampo-retrosplenial communication also involves excitatory circuits ${ }^{14}$. Two major routes carrying hippocampus-related information to the RSCg are a dense corticocortical projection from subiculum, and a subcortical pathway traversing the anterior thalamic nuclei (ATN). Subiculum $\rightarrow$ RSCg projections arise from burst-firing vGlut1- or vGlut2-expressing pyramidal neurons, which directly excite RSCg pyramidal neurons ${ }^{15}$. They also drive feedforward (disynaptic) inhibition ${ }^{15}$, a local-circuit inhibitory mechanism previously implicated in mnemonic functions of the $\mathrm{RSCg}^{12}$. The $\mathrm{ATN} \rightarrow \mathrm{RSCg}$ projections arise particularly from the anteroventral (AV) nucleus, and ramify mainly in $\mathrm{L} 1$ of the $\mathrm{RSCg}^{16,17}$, similar to the GABAergic $\mathrm{CA} 1 \rightarrow \mathrm{RSCg}$ projection. The cellular targets and circuits of $\mathrm{ATN} \rightarrow \mathrm{RSCg}$ axons are unknown, but the anatomical convergence in $\mathrm{L} 1$ of these inhibitory CA1 axons, combined with the presence of apical tuft dendrites of L5 pyramidal (L5pyr) neurons in this layer, suggests the possibility of a highly specific circuit configuration whereby long-range inhibition and thalamocortical (TC) excitation converge to co-regulate RSCg output.

Here, we applied cell-type-specific optogenetic, chemogenetic, electrophysiological, and behavioral tools to address whether retrosplenially projecting CA1 neurons constitute a distinct class of long-range-projecting GABAergic neurons, and to test the hypothesis that they form $\mathrm{CA} 1 \rightarrow \mathrm{RSCg}$ circuits that mediate direct inhibitory hippocampo-retrosplenial interactions, and converge with excitatory ATN-TC inputs via L1 circuit connections to modulate thalamo-retrosplenial interactions. 


\section{Results}

\section{CA1 retrosplenial-projecting (CA1-RP) neurons are a distinct class of GABAergic neurons.}

To characterize the presynaptic neurons in the hypothesized CA1 $\rightarrow$ RSCg circuit, we first anatomically localized them by injecting retrograde tracers into the RSCg of wild-type mice and imaged hippocampal slices (Fig. 1a,b). Labeled neurons were located in dorsal CA1 at the border of stratum radiatum and stratum lacunosum-moleculare (Fig. 1b,c), similar to observations in rats 9,10 . In agreement with prior immunostaining evidence for GABA/ GAD65 expression ${ }^{9}, 10$, we observed $100 \%$ co-labeling of retrograde tracer and mCherry in stratum radiatum/lacunosum-moleculare border neurons in Gad2-mCherry mice (doublelabeling in 62 of 62 tracer-labeled neurons, 6 dorsal hippocampal fields of view, 2 mice), a line with widespread expression in GABAergic neurons (Methods) (Fig. 1d).

Because cellular electrophysiological properties can help to distinguish among GABAergic cell classes, we quantified these based on whole-cell recordings from retrogradely labeled cells in brain slices (Table S1). Voltage responses to current steps revealed a late-spiking pattern at rheobase, and a non-adapting regular spiking pattern above rheobase (Fig. 1e,f). These firing patterns are often, though not uniquely, associated with neurogliaform cells ${ }^{18,19}$.

To explore this possibility, we also characterized the morphological properties of these neurons, by filling them with biocytin during recordings and imaging their dendritic and local axonal arbors (Fig. 1g). Analysis of images and digital reconstructions revealed dendrites extending symmetrically in the horizontal dimension along the stratum radiatum/ lacunosum-moleculare border, but asymmetrically in the radial dimension, with dense branching into stratum radiatum but sparse branching into stratum lacunosum-moleculare (Fig. 1h,i). Conversely, local axons branched primarily in stratum lacunosum-moleculare, not stratum radiatum (Fig. 1h,i). Morphologically, these neurons thus differ markedly from neurogliaform cells described in CA1, which have dendrites localized to stratum lacunosummoleculare and axons that branch profusely near the soma ${ }^{18,20}$.

Because molecular expression patterns are important for defining inhibitory cell classes, we also evaluated these. Confirming Gad2 expression (Fig. 1d), labeled neurons were also observed at the stratum radiatum/lacunosum-moleculare border following injection of a Credependent retrograde virus encoding a fluorescent protein (AAVretro-FLEX-tdTomato) into the RSCg in Gad2-Cre mice (Supplementary Fig. S1a). Consistent with prior results ${ }^{9}$, 10, we observed a lack of expression of parvalbumin, calbindin, calretinin, and somatostatin (Supplementary Fig. S1b). CA1-RP neurons are among several types of interneuron that selectively express the transcription factor COUP-TFII ${ }^{21}$, and many COUP-TFII-positive neurons in stratum radiatum/lacunosum-moleculare are positive for a-actinin and reelin in $\operatorname{rat}^{19,21}$. We found that most CA1-RP neurons were immunopositive for reelin (doublelabeling in 26 of 29 tracer-labeled neurons, 3 dorsal hippocampal fields of view, 2 mice) (Fig. 11 and Supplementary Fig. S1b). As neuropeptide Y (NPY) and neuronal nitric oxide synthase (nNOS) are also important interneuron markers ${ }^{18,19}$, we tested for these and found that most CA1-RP neurons expressed NPY (double-labeling in 24 of 27 tracer-labeled cells, 3 dorsal hippocampal fields of view, 2 mice), but not nNOS (double-labeling in 3 of 20 
tracer-labeled neurons, 3 dorsal hippocampal fields of view, 2 mice) (Fig. 1j,1 and Supplementary Fig. S1b). Lastly, we considered the possibility that the CA1-RP neurons express neuron-derived neurotrophic factor $(\mathrm{Ndnf})^{22}$, as was recently postulated for CA1-RP neurons as well as other retrohippocampal interneurons based on transcriptomic analysis ${ }^{23}$. Following injection of retrograde tracer into the RSCg of Ndnf-Cre mice (crossed with a tdTomato reporter line, Ai14), a majority of the CA1-RP neurons were tdTomato-positive (double-labeling in 50 of 88 tracer-labeled neurons, 11 dorsal hippocampal fields of view from 2 mice) (Fig. 1k,l).

Collectively, these data show that CA1-RP neurons have some properties associated with neurogliaform cells ${ }^{18,19,21}$, but form a long-range axonal projection and also have dendritic and axonal morphology that significantly deviate from defining features of neurogliaform cells. Our characterizations establish the CA1-RP neurons as a distinct class of GABAergic neurons, anatomically positioned to mediate direct inhibitory communication from dorsal hippocampus to RSCg.

\section{CA1-RP neurons form inhibitory synapses onto RSCg-L5pyr neurons at L1 apical tuft dendrites.}

We next investigated the circuits formed by the CA1-RP axonal projections to the RSCg. Following injection of Cre-dependent AAV5-Ef1a-DIO-hChR2-EYFP into CA1 of Gad2Cre mice, labeled neurons were observed in CA1, and labeled axons were observed in L1 of the RSCg (Fig. 2a,b and Supplementary Fig. S2) ${ }^{9,10}$. As a first step in analyzing CA1$\mathrm{RP} \rightarrow \mathrm{RSCg}$ circuits, we used an in vivo approach to ask whether activation of these axons in the intact brain generates a net inhibitory effect on RSCg activity, as expected if connections predominantly involve GABAergic transmission to pyramidal neurons, or other effects, suggesting more complex circuits (e.g. disinhibition). In ketamine-anesthetized mice, we stimulated the ChR2-expressing CA1-RP axons within the RSCg (by delivering brief, 10-ms duration photostimuli via an optical fiber placed directly over the RSCg) while recording multi-unit activity on linear probes placed in L5 of the RSCg (see Methods) (Fig. 2c,d). Photostimulation of CA1-RP axons suppressed RSCg activity, to $62.8 \%$ of baseline levels (multi-unit activity rate exceeded the $-95 \%$ confidence interval of baseline, 15 to $76 \mathrm{~ms}$ poststimulus; baseline activity $7.2 \pm 3.5$ events/s; $\mathrm{n}=8$ mice) (Fig. 2e,f). Thus, these in vivo results show that CA1-RP axons can indeed exert a net inhibitory effect on RSCg activity in the intact network, and point to RSCg-L5pyr neurons as candidate targets of these axons.

For detailed analysis of CA1-RP $\rightarrow \mathrm{RSCg}$ connectivity at the cellular level, we turned to $\mathrm{ex}$ vivo slice-based methods, focusing on L5Pyr neurons. These neurons, which are implicated in mediating diverse functions of the $\mathrm{RSCg}^{12,24-26}$, are a prominent source of apical tuft dendrites in $\mathrm{L1}^{27}, 28$ (Fig. 2g). Thus, the apparent overlap in L1 of CA1-RP axons and apical tuft dendrites of L5pyr neurons implies an anatomical basis for monosynaptic GABAergic connections. However, these pre- and postsynaptic elements could in fact have minimal actual overlap, due, for example, to fine-scale micromodularities such as dendritic bundling and patchy axonal arborizations ${ }^{29}$. Moreover, and more fundamentally, axo-dendritic overlap only indicates potential connectivity, and does not reliably predict actual connectivity (reviewed in ${ }^{30,31}$ ). We therefore used an optogenetic-electrophysiological strategy to test 
and characterize physiological synaptic connections from presynaptic CA1-RP axons to postsynaptic RSCg-L5pyr neurons. Recordings in acute brain slices from L5pyr neurons during wide-field photostimulation of ChR2-expressing CA1-RP axons showed outward inhibitory postsynaptic currents (IPSCs) at a command voltage of $\sim 10 \mathrm{mV}$, but no inward excitatory postsynaptic currents (EPSCs) at $-70 \mathrm{mV}$ (Fig. 2h,i), consistent with purely GABAergic transmission. Indeed, IPSCs remained after blocking fast glutamatergic transmission (with NBQX and CPP), and after isolation of purely monosynaptic inputs (with TTX and 4-AP) ${ }^{32}$, but were abolished by blocking fast GABAergic transmission (before vs after SR-95531: $31.7 \pm 8.7$ vs $0.3 \pm 0.3 \mathrm{pA} ; \mathrm{n}=7, \mathrm{p}=0.016$, signed-rank test) (Fig. $2 \mathrm{~h}$ ). Blocking $\mathrm{GABA}_{\mathrm{B}}$ receptors caused a small reduction in IPSC amplitudes (Supplementary Fig. S3). In current clamp recordings, focal laser stimulation of CA1-RP axons in L1 (in drug-free conditions; see Methods) evoked inhibitory postsynaptic potentials in RSCg-L5pyr neurons that arrived and peaked with short latencies $(5.2 \pm 0.5 \mathrm{~ms}$ latency from stimulus onset to response onset, based on $10 \%$ of the peak inhibitory response; $15.8 \pm 1.0 \mathrm{~ms}$ latency to peak inhibition; $\mathrm{n}=7$ neurons), a temporal profile resembling the suppressive effect observed in vivo (Fig. 2f, inset). Thus, these results show that, at the cellular level, CA1-RP neurons form monosynaptic, $\mathrm{GABA}_{\mathrm{A}}$-mediated, fast inhibitory synaptic connections to RSCg-L5pyr neurons.

At the subcellular level, we wished to resolve which dendritic compartments of the L5pyr neurons receive CA1-RP input, as these can differ greatly in their computational and plasticity properties ${ }^{33,34}$. The anatomical overlap of CA1-RP axons with the apical tuft dendrites of L5pyr neurons in L1 suggests that the IPSCs might arise from inhibitory synapses at this location, despite electrotonic attenuation and imperfect space-clamp. However, a plausible alternative possibility is that the observed IPSCs could instead or additionally reflect strong perisomatic synapses. To localize the dendritic sites of CA1-RP synapses onto L5pyr neurons, we used a subcellular mapping technique, sCRACM (Methods, Fig. 2j). Subcellular input maps showed that functional inhibitory synaptic contacts were localized to apical tuft dendrites in L1 (mean input to apical vs basal dendrites: $565.4 \pm 108.3$ vs $164.3 \pm 25.4$ pA; $\mathrm{n}=21$ neurons, $\mathrm{p}=8 \mathrm{e}-05$, signed-rank test; Fig. $2 \mathrm{k}-\mathrm{m}$ ). These results reveal a novel apical tuft-targeting inhibitory innervation pattern, one not previously demonstrated by unbiased mapping but consistent with the anatomical axo-dendritic overlap of presynaptic CA1-RP axons in L1 and postsynaptic apical tuft dendrites of L5pyr neurons.

We performed additional studies to investigate dynamic aspects of $\mathrm{CA} 1-\mathrm{RP} \rightarrow \mathrm{RSCg}-\mathrm{L} 5 \mathrm{pyr}$ connections. In one set of experiments, we assessed the short-term plasticity of these synapses, as activity-dependent changes in synaptic efficacy are important mechanisms involved in network dynamics 35,36 . To avoid potential artifacts associated with wide-field illumination of ChR2-expressing presynaptic terminals, we used a laser to focally stimulate axons away from postsynaptic dendrites. Trains of IPSCs evoked by repetitive laser stimulation of CA1 axons in L1 (in drug-free conditions; see Methods for details) displayed strong synaptic depression (Supplementary Fig. S4). In other experiments we found that these apical dendritic inhibitory inputs were sufficiently potent, despite their electrotonic remoteness, to prolong inter-spike intervals during trains of action potentials in the L5pyr neuron evoked by current injection (see Methods; Supplementary Fig. S5). These results 
demonstrate that, on a millisecond time scale, and consistent with the in vivo results presented above, activation of monosynaptic CA1-RP inhibitory input is capable of briefly suppressing activity of RSCg-L5pyr neurons. However, due to synaptic depression, the strength of this inhibitory influence likely diminishes during repetitive activity.

Collectively, these analyses of CA1-RP $\rightarrow \mathrm{RSCg}$-L5pyr circuits show that CA1-RP axons form monosynaptic $\mathrm{GABA}_{\mathrm{A}}$-mediated inhibitory synapses onto RSCg-L5pyr neurons, via depressing synapses made primarily onto apical dendritic tufts, capable of exerting a rapid, inhibitory effect on RSCg activity.

\section{ATN-TC axons form excitatory synapses onto RSCg-L5pyr neurons at L1 apical tuft dendrites.}

In addition to these direct inhibitory projections from CA1, hippocampus communicates with RSCg via pathways involving the anterior thalamic nuclei (ATN), particularly the anteroventral (AV) nucleus ${ }^{37,38}$, which sends a thalamocortical (TC) projection ramifying mainly in $\mathrm{L} 1$ of $\mathrm{RSCg}^{16}$ - anatomically strikingly similar to that of CA1-RP axons, but presumably having an opposite, excitatory effect. To test this, we characterized ATN-TC connectivity to RSCg-L5pyr neurons, using a similar multi-step approach as for the CA1 projection.

First, we injected the RSCg in wild-type mice with tracer to retrogradely label TC neurons in thalamus, which verified that AV is the major source of its thalamic afferents (Fig. 3a) ${ }^{17}$. Then, we injected AV with AAV1-CamKIIa-hChR2-mCherry to anterogradely label TC axons, which indicated that in mice these axons indeed target primarily L1 in RSCg, and particularly L1a (its outermost sublayer), with additional sparser branching in deeper layers and in dysgranular RSC (Fig. 3b). To further assess if these L1 axons in RSCg arise from AV neurons, which may branch in other layers ${ }^{16}$, and/or from TC neurons in surrounding nuclei such as the anterodorsal nucleus ${ }^{17,39}$, we repeated this experiment using Grp_KH288-Cre mice, a driver line showing Cre-expressing neurons in a subset of $\mathrm{AV}$ nucleus neurons but none in the anterodorsal nucleus (Supplementary Fig. S6a-h). Following injection of AV with Cre-dependent AAV5-Ef1a-DIO-hChR2-EYFP, we again observed labeled axons in L1 (Fig. 3c,d and Supplementary Fig. S6i-m), supporting the results obtained with wild-type mice indicating that $\mathrm{AV}$, rather than the anterodorsal nucleus, is the main source of L1targeting projections from the ATN to the RSCg. Since targeted injections into AV in wildtype mice resulted in more extensive labeling of AV-TC neurons (than in the Cre line), we used this approach for further study of these AV-predominant ATN $\rightarrow$ RSCg projections.

Next, we assessed the impact of ATN-TC input on RSCg activity in the intact brain using the same in vivo approach as for the CA1-RP inputs (see above, and Methods), recording with linear arrays to sample multi-unit activity in L5 of RSCg while stimulating ChR2-expressing $\mathrm{TC}$ axons (labeled by injecting AAV-ChR2 in wild-type mice) by delivering brief photostimuli via an optical fiber placed directly over the RSCg. Photostimulation of ATNTC axons rapidly and sharply increased RSCg activity above baseline levels (multi-unit activity rate exceeded the $+95 \%$ C.I. of baseline, $\sim 3$ to 27 ms poststimulus; baseline activity $7.5 \pm 2.8$ events/s; $\mathrm{n}=6$ mice) (Fig. 3e), followed by suppression (activity exceeded the $-95 \%$ C.I. of baseline, $\sim 31$ to 58 ms poststimulus). Thus, these in vivo results show that 
ATN-TC axons can indeed exert a brief, net excitatory effect on RSCg activity in the intact network, and point to RSCg-L5pyr neurons as candidate targets of these axons.

We then assessed ATN $\rightarrow$ RSCg synaptic connectivity at the cellular level, using slice-based methods as for the CA1-RP connections (see above, and Methods). Wide-field photostimulation of ATN-TC axons in RSCg slices generated EPSCs in L5pyr neurons, sampled at a command voltage of $-70 \mathrm{mV}$. At command voltage of $\sim 10 \mathrm{mV}$, short-latency IPSCs could also be detected, suggesting feedforward (e.g. disynaptic) inhibition (Supplementary Fig. S7a,b). Confirming this, only the inward postsynaptic current remained after bath application of TTX and 4-AP (Supplementary Fig. S7a), indicating that the EPSCs are monosynaptic and the IPSCs are disynaptic. Application of blockers of glutamatergic transmission (NBQX and CPP) abolished the EPSCs (before vs after drugs: $-7.1 \pm 2.0$ vs $-0.5 \pm 0.4 \mathrm{pA} ; \mathrm{n}=5$ neurons, $\mathrm{p}=0.01,2$-tailed paired t-test), indicating that ATN-TC axons provide monosynaptic glutamatergic transmission to L5pyr neurons (Fig. 3f). In current clamp recordings, focal laser stimulation of ATN-TC axons in L1 evoked excitatory postsynaptic potentials in RSCg-L5pyr neurons that arrived and peaked with short latencies $(4.5 \pm 0.4 \mathrm{~ms}$ latency from stimulus onset to response onset, based on $10 \%$ of the peak excitatory response; $9.0 \pm 1.3$ ms latency to peak excitation; $\mathrm{n}=7$ neurons), a temporal profile resembling the excitatory effect observed in vivo (Fig. 3e, inset). Thus, these results show that, at the cellular level, ATN-TC neurons form monosynaptic, glutamatergic, excitatory synaptic connections to RSCg-L5pyr neurons.

We further analyzed ATN-TC $\rightarrow$ RSCg-L5pyr connections to localize the dendritic sites of input and assess dynamic properties. Mapping the subcellular location of ATN-TC inputs (by sCRACM; see above, and Methods) showed that, on average, ATN-TC inputs targeted the apical tuft dendrites of L5pyr neurons (mean input to apical vs basal dendrites: -103.2 \pm 25.5 vs $-26.2 \pm 12.5$ pA; $n=17$ neurons, $\mathrm{p}=0.002$, signed-rank test; Fig. $3 \mathrm{~g}-\mathrm{i})$. In separate experiments, evaluating the short-term plasticity of these synapses (using repetitive laser stimulation aimed at the ATN axons in L1; see above, and Methods) showed that trains of EPSCs displayed a pattern of non-depressing initial responses followed by depressing later responses (Supplementary Fig. S4). In other experiments, current-clamp recordings from RSCg-projecting AV neurons showed that these displayed expected patterns of membrane potential-dependent burst-firing ${ }^{40}$ (Supplementary Fig. S7c). Results from these experiments thus add important information about synaptic localization and dynamic signaling in ATN-TC $\rightarrow$ RSCg-L5pyr circuits.

Given that CA1-RP and ATN-TC axons both target L1, and both monosynaptically innervate L5pyr neurons at their apical tuft dendrites, do individual RSCg-L5pyr neurons receive both ATN-TC and CA1-RP inputs? To test this, we co-stimulated ChR2-expressing CA1 and ATN axons in L1 and measured post-synaptic potentials (PSPs) before and after blocking CA1 inputs with SR-95531, with TTX/4-AP in the bath and $\mathrm{Cs}^{+}$-based internal solution (see Methods for details). Overall, the photo-evoked PSP became significantly more positive after removal of CA1 inputs (before vs after $10 \mu \mathrm{M}$ SR-95531: $-0.20 \pm 0.17$ vs $0.60 \pm 0.21$ $\mathrm{mV} ; \mathrm{n}=10$ neurons, $\mathrm{p}=0.002$, signed-rank test (Fig. $3 \mathrm{j}, \mathrm{k})$. These data indicate that the two types of input tend to target a common pool of RSCg-L5pyr neurons in a convergent manner, rather than forming parallel pathways innervating separate pools. 
Another source of excitation to RSCg-L5pyr neurons is from pyramidal neurons in subiculum, which innervate pyramidal neurons in both superficial and deep layers of $\mathrm{RSCg}^{15}$. Although subicular axons project densely to L3 of RSCg, they also ramify sparsely in other layers (Fig. 31); it is therefore uncertain which dendritic compartments of L5pyr neurons they excite. We injected AAV1-CamKIIa-hChR2-mCherry into subiculum to label excitatory neurons (Supplementary Fig. S8), and used SCRACM to map the dendritic locations of these subicular inputs, since this can help to understand how postsynaptic mechanisms integrate all three inputs (CA1, thalamus, and subiculum). Subicular inputs mainly innervated basal dendrites of L5pyr neurons, with relatively weak input to apical tuft dendrites in L1 (mean input to apical vs basal dendrites: $-84.9 \pm 16.9$ vs $-157.2 \pm 31.2 \mathrm{pA}$; $\mathrm{n}=26$ neurons, $\mathrm{p}=0.016$, signed-rank test; Fig. $3 \mathrm{~m}, \mathrm{n})$. Thus, the input pattern differed from that expected based on axo-dendritic overlap, unlike CA1-RP and ATN-TC inputs. In separate experiments we characterized the short-term plasticity of these synapses, which showed a pattern of facilitating responses (Supplementary Fig. S4). We previously characterized the spiking patterns of the presynaptic RSCg-projecting neurons in the subiculum as burst-firing ${ }^{15}$. These results indicate that subicular axons target a more proximal subcellular dendritic compartment and express a facilitating pattern of short-term plasticity compared to CA1 and thalamic inputs to L5pyr neurons.

Overall, these findings show that ATN-TC axons form monosynaptic glutamatergic connections onto RSCg-L5pyr neurons that target apical tuft dendrites and can exert a rapid, excitatory effect on RSCg activity. Together with the previous results, this indicates that ATN-TC projections and CA1-RP projections form a convergent triadic circuit with RSCgL5pyr neurons, with anatomical alignment of afferent input at the cellular and subcellular levels, and opposing actions.

\section{Opposing actions of CA1-RP inhibition and ATN-TC excitation in contextual fear learning.}

The anatomical convergence of $\mathrm{CA} 1-\mathrm{RP} \rightarrow \mathrm{RSCg}$ and $\mathrm{ATN}-\mathrm{TC} \rightarrow \mathrm{RSCg}$ projections onto the apical tuft dendrites of L5pyr neurons combined with the physiologically opposing actions of neurotransmission suggests that these circuit connections contribute differentially to the functions of the RSCg in processing hippocampal-dependent memories. To explore this, we used contextual fear conditioning (CFC), a behavioral paradigm that induces hippocampaldependent contextual fear memory in mice and modulates RSC activity (Methods).

To test the roles of each pathway in memory encoding, we selectively silenced presynaptic axons from either source at their arborizations within the RSCg during CFC, using a previously established chemogenetic approach based on expressing $\mathrm{hM} 4 \mathrm{D}(\mathrm{Gi})$ in presynaptic neurons and blocking synaptic release from a subset of axonal branches by local infusion of clozapine-N-oxide (CNO) (Methods; Fig. 4a). As in prior studies using this approach ${ }^{15}$ we performed control experiments to test the efficacy of the technique, which confirmed that $\mathrm{CNO}$ application reduced synaptic transmission in both circuits (Supplementary Fig. S9).

Mice underwent bilateral injections of virus (Cre-dependent or -independent AAV$\mathrm{hM} 4 \mathrm{D}(\mathrm{Gi})$, as appropriate) into either CA1 (Gad2-Cre mice) or AV (wild-type mice), followed by bilateral implantation of cannulae in RSC, which were used to infuse either 
CNO (CNO group) or vehicle (vehicle group) 30 min before CFC. We have previously shown that infusion of $\mathrm{CNO}$ alone into the RSC of wild-type mice (i.e., without hM4D(Gi) expression) did not induce effects during $\mathrm{CFC}^{15}$. In additional control experiments, adding a fluorescent dye to the infusant showed that infusions remained in the RSC, with little or no detectable spread to subiculum or CA1 (Methods; Supplementary Fig. S91-p; see also ref. ${ }^{15}$ ). Silencing of CA1-RP axons in the RSCg during CFC increased freezing behavior during the retrieval test in the CNO-treated group compared to controls (mean freezing, vehicle vs CNO group: $45.6 \pm 5.0 \%$ vs $64.1 \pm 4.9 \% ; n=14$ vs 9 mice, $p=0.03$, rank-sum test; Fig. 4b). CNO did not affect activity in the conditioning box during training or activity burst to the shock (mean activity during training, vehicle vs CNO group: $9.5 \pm 1.9 \mathrm{~cm} / \mathrm{s}$ vs $11.8 \pm 2.5$ $\mathrm{cm} / \mathrm{s} ; \mathrm{p}=0.46$, rank-sum test; mean activity burst to the shock: $25.6 \pm 1.9 \mathrm{~cm} / \mathrm{s}$ vs $30.3 \pm 8.4$ $\mathrm{cm} / \mathrm{s} ; \mathrm{p}=0.69$, rank-sum test). In contrast, silencing of ATN-TC axons during CFC caused a significant reduction in freezing behavior in retrieval test in the CNO-treated group compared to controls (mean freezing, vehicle vs CNO group: $62.9 \pm 3.4 \%$ vs $37.1 \pm 7.2 \%$; $\mathrm{n}$ $=17$ vs 10 mice, $\mathrm{p}=0.009$, rank-sum test; Fig. $4 \mathrm{c}$ ). Again, $\mathrm{CNO}$ did not affect activity in the conditioning box during training or activity burst to the shock (mean activity during training, vehicle vs CNO group: $15.4 \pm 1.2 \mathrm{~cm} / \mathrm{s}$ vs $19.5 \pm 2.0 \mathrm{~cm} / \mathrm{s} ; \mathrm{p}=0.08$, rank-sum test; mean activity burst to the shock: $57.7 \pm 2.8 \mathrm{~cm} / \mathrm{s}$ vs $59.0 \pm 4.5 \mathrm{~cm} / \mathrm{s} ; \mathrm{p}=0.86$, rank-sum test). These data indicate that CA1-RP and ATN-TC circuits are both engaged during the encoding of contextual fear memory, but appear to serve opposing roles, with the inhibitory pathway normally suppressing and the excitatory pathway enhancing the expression of context memories.

\section{Discussion}

Harnessing the availability of an array of tools for targeted analysis of long-range circuits in the mouse, we dissected the synaptic connectivity mediating the confluence and interaction of major afferent projections to the RSCg from dorsal hippocampus and ATN. Our findings provide cellular- and subcellular-level mechanistic insight into information-processing in these hippocampo-thalamo-cortical networks (Fig. 5).

Our characterizations of CA1-RP neurons, the source of the CA1 inhibitory projection to $\mathrm{RSCg}$, reveal a unique combination of properties defining them as a distinct GABAergic cell class. Certain properties, such as firing patterns and molecular expression, resemble those described in various types of local interneurons, particularly neurogliaform cells. However, they exhibit unusual features not shared with neurogliaform cells, including asymmetric dendritic morphology and mostly $\mathrm{GABA}_{\mathrm{B}}$-independent synaptic transmission. In addition, although their axons innervated apical tuft dendrites in L1 like local neurogliaform cells $^{20,41}$, they did so through long-range projections. We also observed variability in marker labeling (e.g. Ndnf), suggesting some degree of within-class heterogeneity. The results of our characterizations appear consistent with a recent single-cell transcriptomic analysis that assigns retrohippocampally projecting CA1 interneurons, including putative CA1-RP neurons, to a cluster of cells - the Ntng1.Rgs10 subgroup in "continent 6" - that form long-range inhibitory projections and lack expression of most classical interneuron markers ${ }^{23}$. Our results thus help to define the cellular identity and basic characteristics of these long-range-projecting, apical tuft-targeting, CA1-RP inhibitory neurons. 
Optogenetic-electrophysiological circuit analyses showed that CA1-RP axons directly inhibited the apical tuft dendrites of RSCg-L5pyr neurons, converging there with excitatory connections from ATN-TC neurons, while excitatory subicular inputs mainly innervate the perisomatic dendrites in deeper layers (Fig. 5). This circuit configuration bears close resemblance to generalized cortical circuit models in which L5pyr neurons receive dual excitatory inputs to their apical tuft dendrites via L1 (e.g. from matrix thalamus) and to proximal dendrites via deeper layers (e.g. corticocortical), which synergistically drive firing 33,42 . The essential concept is that when both input channels are active they are associatively paired, through active dendritic conductances, resulting in robust firing of the postsynaptic pyramidal neuron ${ }^{33}$. Similar circuits and pairing mechanisms have been identified in hippocampus, where coincident activation of perforant-path and CA3 inputs to apical and proximal dendrites of CA1 pyramidal neurons drives non-linear responses and synaptic plasticity ${ }^{43}$. Importantly, the associative pairing mechanism can be suppressed by local apical tuft-targeting interneurons ${ }^{33}$. The RSCg circuits delineated here appear remarkably analogous - with the extraordinary variation that the apical tuft inhibition is supplied remotely, by CA1-RP neurons. To the extent that the universal model proposed by Larkum ${ }^{33}$ holds for these RSCg circuits, a clear implication is that one function of CA1$\mathrm{RP} \rightarrow \mathrm{RSCg}$ inhibition is to suppress the associative pairing, by RSCg-L5pyr neurons, of information carried by thalamic and subicular afferents.

$\mathrm{CA} 1-\mathrm{RP} \rightarrow \mathrm{RSCg}$ circuits are likely to have multiple other functional roles as well. Some are suggested by analogy to functions ascribed to local apical tuft-targeting interneurons in neocorte ${ }^{44}$, including modulation of sensory input-driven dendritic events ${ }^{41,45}$, and the promotion of "robustness" by reducing the variability of cortical neuronal responses to sensory inputs ${ }^{46}$. In this way, CA1-RP inhibition might contribute to the "sharpening" of contextual memory representations ${ }^{47}$. By analogy to GABAergic projections from zona incerta to neocortex, which similarly ramify in L1 and inhibit L5pyr neurons ${ }^{3}$, CA1-RP neurons might be important for cortical development, or for braking excitation to prevent epileptiform activity. They may be involved in oscillations and rhythmogenesis, by analogy to long-range inhibitory projections in septo-hippocampal networks ${ }^{48}$. The behavioral roles of CA1-RP neurons' circuits may be similarly protean. Chemogenetic disconnection of the inhibitory CA1 or excitatory ATN inputs to RSCg gave opposing effects on one aspect of behavior, the encoding of fear memory. However, while this accords with the opposing neurotransmitter actions of these pathways, the contributions of these circuits to other aspects of RSCg-dependent behavior are likely more complex. Nevertheless, our results, together with our recent findings demonstrating contributions of excitatory subiculum $\rightarrow$ RSCg pathways to contextual fear conditioning ${ }^{15}$, suggest a role for the inhibitory CA1-RP $\rightarrow \mathrm{RSCg}$ projection in modulating the impact of thalamic inputs relative to that of subicular inputs, perhaps to avoid inappropriate and potentially pathological behavioral responses due to unregulated associative pairing of thalamic and subicular inputs. Indeed, dysfunction in RSCg-related pathways has been implicated in a variety of neurological and psychiatric disorders ${ }^{13}$, as has dysfunction in TC circuits $^{37,49}$. Our findings thus additionally present a detailed framework for investigating CA1-RP neurons and RSCg circuits as etiological factors in these conditions, and as targetable cellular candidates for novel therapeutic interventions. 
Our findings also raise many further questions about the hodology and physiology of these and related circuits. For the CA1-RP neurons in particular, which other excitatory and inhibitory RSCg neurons are - and are not - postsynaptic targets of their axons? To what extent do their axons branch elsewhere, such as subiculum ${ }^{10}$ ? What are their local and longrange presynaptic sources of excitatory and inhibitory input? Are CA1-RP inputs involved in coordinating rhythmic activity in the two areas? Future studies can build on our results to further illuminate the cellular mechanisms underlying hippocampo-thalamo-retrosplenial interactions.

\section{Online methods}

\section{Animal care and mouse lines.}

Studies were approved by Northwestern University Animal Care and Use Committee and followed the animal welfare guidelines of the National Institutes of Health. All experiments were performed using mouse strain C57BL/6 or transgenic mice with C57BL/6 background. Male or female mice were used in approximately equal numbers. Adult mice ( $>2$ months) were used for all experiments, and were 9-20 weeks old at the time of electrophysiological and behavioral studies. Mice were kept on a 12-hour light/dark cycle and had unrestricted access to the food and water.

Gad2-Cre (RRID:IMSR_JAX:010802) $)^{50}$. Gad2-mCherry (RRID:IMSR_JAX:023140) ${ }^{51}$, and Ndnf-Cre (RRID:IMSR_JAX:028536) ${ }^{22}$ mice were obtained from Jackson Laboratory. Grp_KH288-Cre mice (RRID:MMRRC_037585-UCD) ${ }^{52}$ were obtained from MMRRC, and back-crossed in-house with C57BL/6 mice for at least 6 generations. All Cre lines were maintained by crossing with C57BL/6 mice. Experiments were performed using heterozygous mice, identified to be positive for Cre with genotyping. Gad2-mCherry mice were maintained by homozygous breeding. In some cases, heterozygous litters of each Cre lines were crossed with tdTomato reporter line Ai14 from Jackson laboratory (RRID:IMSR_JAX:007908).

\section{Viral vectors.}

For Cre-dependent anterograde expression of ChR2 and hM4D(Gi), AAV5-Ef1a-DIOhChR2(E123T/T159C)-EYFP-WPRE-hGH (Addgene 35509) and AAV8-hSyn-DIOhM4D(Gi)-mCherry (Addgene 44362) were used. For Cre-independent anterograde expression of ChR2 and hM4D(Gi), AAV1-CamKIIa-hChR2(E123T/T159C)-mCherryWPRE-hGH (Addgene 35512) and AAV8-hSyn-HA-hM4D(Gi)-mCherry (Addgene 50475) were used. For Cre-dependent retrograde infection with AAVretro ${ }^{53}$, AAVrg-CAG-FlextdTomato-WPRE (Addgene 51503), was used. Viral vectors were purchased from University of Pennsylvania Viral Vector Core or Addgene.

\section{In vivo stereotaxic injections.}

Mice were anesthetized with isoflurane, head-fixed to a stereotaxic frame, and thermally supported with a feedback-controlled heating pad (DC Temperature Control System, FHC). Buprenorphine $(0.3 \mathrm{mg} / \mathrm{kg})$ and meloxicam $(1 \mathrm{mg} / \mathrm{kg})$ were injected subcutaneously for post-operative pain relief. After incising the scalp over the cranium, small craniotomy was 
opened using a dental drill over the RSC, dorsal CA1 of the hippocampus, or the AV nucleus of the thalamus. The stereotaxic coordinates of the RSCg target were (relative to bregma, in $\mathrm{mm}$ ) anteroposterior -1.6 and 2.0, lateral 0.1 , and ventral 0.3, 0.6 and 0.9, CA1 target were anteroposterior -2.0, lateral 1.5, and ventral 1.4, and those of the AV target were anteroposterior -0.5 , lateral 1.2, and ventral 2.8. A beveled injection pipette, back-filled with mineral oil and front-filled with AAV solution, was slowly advanced to the target depth, where a small volume $(50 \mathrm{~nL})$ of virus was injected using a displacement-driven injector (MO-10 Narishige). The pipette was left in place for 5 mins before retraction. Once retracted, the incision was closed with a nylon or silk suture.

\section{Slice electrophysiology.}

Mice were euthanized 3-5 weeks after virus injection and coronal brain slices ( $250 \mu \mathrm{m}$ thick for RSCg and AV, $300 \mu \mathrm{m}$ thick for CA1) were prepared using a vibratome (VT1200S, Leica) in ice-cold choline-based cutting solution (composition, in mM: $25 \mathrm{NaHCO}_{3}, 1.25$ $\mathrm{NaH}_{2} \mathrm{PO}_{3}, 2.5 \mathrm{KCl}, 0.5 \mathrm{CaCl}_{2}, 7 \mathrm{MgCl}_{2}, 110$ choline chloride, 11.6 sodium L-ascorbate, and 3.1 sodium pyruvate). The rostral end of the brain was first removed by a coronal blocking cut. The remaining part of the brain was mounted caudal-end-up on the exposed surface. Slices were collected in a caudal-to-rostral manner, starting with the first slice in which the corpus callosum was observed and proceeding for four more slices, spanning the RSCg. Recordings were mostly made from the middle three slices in this series. Slices were transferred to artificial cerebrospinal fluid (ACSF; composition, in mM: $127 \mathrm{NaCl}, 25 \mathrm{D}$ glucose, $2.5 \mathrm{KCl}, 1 \mathrm{MgCl}_{2}, 2 \mathrm{CaCl}_{2}$, and $1.25 \mathrm{NaH}_{2} \mathrm{PO}_{3}$ ) for 30 minutes at $34{ }^{\circ} \mathrm{C}$ and then 1 hour or more at room temperature $\left(\sim 21^{\circ} \mathrm{C}\right)$ before recording. Whole-cell recordings were performed using an upright microscope (BX51WI, Olympus) equipped with gradientcontrast and epifluorescence optics. Pipettes ( 2.5-4 M $\Omega$ ) were filled with either cesium- or potassium-based internal solution containing (in $\mathrm{mM}$ ): 128 potassium or cesium methanesulfonate, 10 HEPES, 10 phosphocreatine, $4 \mathrm{MgCl}_{2}$, 4 ATP, 0.4 GTP, and 3 ascorbate, $\mathrm{pH}$ 7.25, 290-295 mOsm. For cesium-based solution, $1 \mathrm{mM} \mathrm{QX-314} \mathrm{and} 1 \mathrm{mM}$ EGTA was also included. To visualize neurons after the recording, $0.05 \mathrm{mM}$ Alexa 647 hydrazide and/or biocytin $(4 \mathrm{mg} / \mathrm{mL})$ was added to internal solution. Using a $60 \times$ objective lens (LUMPlanFI/IR, N/A 0.9, Olympus), recordings were targeted either to L5pyr neurons (RSCg slices) or fluorescently labeled RSCg-projecting neurons in CA1 (hippocampal slices). ACSF, oxygenated with $95 \% \mathrm{O}_{2} / 5 \% \mathrm{CO}_{2}$, was perfused using a pump-driven recirculation system, and the recording temperature was maintained at $32{ }^{\circ} \mathrm{C}$ (most experiments) or $34^{\circ} \mathrm{C}$ (intrinsic-property recordings) using an in-line temperature controller (TC-324B, Warner instrument). Data were acquired using Ephus software ${ }^{54}$. Signals were amplified with an Axon Multiclamp 700B (Molecular Devices), filtered at $4 \mathrm{kHz}$, and sampled at $10 \mathrm{kHz}$ (most experiments) or $40 \mathrm{kHz}$ (intrinsic-property recordings).

\section{Wide-field ChR2 photostimulation.}

Wide-field photostimulation was performed as described previously ${ }^{55}$. Brief $(5 \mathrm{~ms})$ flashes from a blue LED $\left(1 \mathrm{~mm}^{2} / \mathrm{mW}\right.$, M470L3, Thorlab) were delivered through a $4 \times$ objective lens (UPlanSApo, N/A 0.16, Olympus) focused onto the specimen, by gating the LED output with a TTL signal. Trials were repeated at $10 \mathrm{~s}$ intervals. Recordings were made in voltage-clamp mode and drug-free conditions, with the command potential set to $-70 \mathrm{mV}$ to 
record EPSCs. It was then changed to $0-10 \mathrm{mV}$ to record IPSCs from same neuron. Tetrodotoxin (TTX, $1 \mu \mathrm{M}$ ) and 4-aminopyridine (4-AP, $100 \mu \mathrm{M})$ were added to isolate monosynaptic connections ${ }^{32}$. Traces were analyzed offline by averaging $3-5$ trials per condition, baseline-subtracting based on the $100 \mathrm{~ms}$ pre-stimulus, and calculating the mean amplitude over the time window 0 to $50 \mathrm{~ms}$ from stimulus onset.

\section{Subcellular ChR2-assisted circuit mapping (sCRACM).}

SCRACM was performed as described previously ${ }^{32,56}$ using a laser scanning photostimulation system consisting of a blue laser (model MLL-FN-473, $473 \mathrm{~nm}, 50 \mathrm{~mW}$, CNI Laser), electro-optical modulator (model 350-50, Conoptics), mechanical shutter (model VMM-D1, Uniblitz), and mirror galvanometers (model 6210, Cambridge Technologies). The laser beam was focused through a $4 \times$ objective lens onto the slice ( 60 $\mu \mathrm{m}$ diameter, full-width at half maximum). Laser power was monitored using a photodiode (part number 53-379, silicon detector, blue enhanced response, $100 \mathrm{~mm}^{2}$, Edmund Optics) and adjusted by a graded neutral density filter to $0.75 \mathrm{~mW}$ at the specimen. An image of the slice was captured using the $4 \times$ objective lens, oriented with the pia up, and overlaid with a graphical representation of the photostimulation grid (16 by 16 square array with $50 \mu \mathrm{m}$ spacing), which was rotated and offset to align its top row with the pia and its middle columns with the soma. Each site was stimulated in pseudo-random order at an interstimulus interval of 0.4 or $1 \mathrm{~s}$. Mapping was repeated 3 times for each neuron, using a different pseudo-random sequence each time. In experiments involving $\mathrm{GABA}_{\mathrm{B}}$ pharmacology (Supplementary Fig. S3) and current-clamp recordings (insets of Fig. 2f, 3e), the laser scanning system was used to photostimulate axons at a single spot in L1.

\section{Repetitive stimulation of axons.}

Brain slices were prepared from CA1-, AV-, or subiculum-injected mice, as described above. Whole-cell recordings were performed in voltage-clamp mode with $\mathrm{Cs}^{+}$-based internal solution, in drug-free conditions (plain ACSF, without TTX/4-AP), as described above. Sites in L1 (for CA1 and ATN axons) or deeper layers (L3/5, for subicular axons), approximately $\sim 250$ um lateral to either side of soma, were selected for targeted focal photostimulation, to avoid potential artifacts associated with direct stimulation of ChR2-expressing axon terminals ("over-bouton stimulation") ${ }^{57}$. Stimulus intensity was set to $\sim 0.75 \mathrm{~mW}$ at the focal plane. The laser beam was flashed (1 ms duration) at the selected sites in a sequential manner ( $1 \mathrm{~s}$ inter-stimulus interval), to identify a site of input. If no responses were observed, new sites were tested. Once a suitable hotspot of input was located, axons at this site were repetitively stimulated with a train of 10 pulses (1-ms duration each), at $20 \mathrm{~Hz}$. Control experiments showed that action potentials were reliably evoked during repetitive stimulation using these parameters ( $n=7$ neurons, Supplementary Fig. S4). Ten sweeps were collected, and traces were analyzed offline to characterize mean response amplitudes and short-term dynamics.

\section{Ex vivo analysis of convergent inputs.}

In this case, both CA1 and AV were injected (with Cre-dependent and Cre-independent AAV-ChR2, respectively, as described above) in the same animals (Gad2-Cre mice), to express ChR2 in both input pathways to the RSCg. Methods were as described above for 
each input pathway separately. Optogenetic photostimulation and electrophysiological recordings were made in slices as described above, with TTX/4-AP in the bath solution to prevent disynaptic responses. The laser beam was targeted to L1, and brief (1 ms) photostimuli were delivered to activate $\mathrm{ChR} 2$-expressing axons and their presynaptic terminals synapsing onto the recorded L5pyr neurons. After sampling pre-drug responses under control conditions, SR-95531 was added to the bath $(10 \mu \mathrm{M})$, and post-drug responses were collected.

\section{2-photon microscopy.}

Slices with biocytin-filled neurons were fixed in 4\% PFA (in 0.1M PBS) overnight, then washed in PBS before incubating in PBS containing 2\% Triton X-100 (Sigma) and streptavidin conjugated with Alexa568 (1:200, Invitrogen) for at least 14 hours at $4{ }^{\circ} \mathrm{C}$. Slices were washed in PBS the next day and mounted onto a glass coverslip (\#1.5, $22 \times 40$ $\mathrm{mm}$, Warner Instruments), imaging side down. A well for the slice was crafted from two 1.5 $\mathrm{mm}$ thick glass coverslip (\#1, $22 \times 22 \mathrm{~mm}$, Warner), which were stacked and attached to the short edges of the main coverslip. After removing excess PBS from the slice, drops of mounting media (FluorSave, Millipore) were applied before sealing with glass slide $(75 \times 25$ $\times 1 \mathrm{~mm}$, Ever Scientific). Once the mounting media solidified, the slide was sealed with nail polish.

Two-photon imaging was performed on a Nikon A1R MP+ Multiphoton system using a water-immersion $25 \times$ objective lens (APO LWD, N/A 1.10, WD $2.0 \mathrm{~mm}$, optimized for coverglass thickness $0.17 \mathrm{~mm}$, Nikon) with the laser (Ti:sapphire, Chameleon Vision, Coherent) tuned to $860 \mathrm{~nm}$ wavelength. Image stacks were acquired using Nikon NISElements software, with settings of at $1024 \times 1024$ pixels $(0.5 \mu \mathrm{m} x-y$ pixel size $), 2.4 \mu \mathrm{s}$ dwell time, and $2 \mu \mathrm{m}$ z-step size. The laser power and the gain of the GaAsP photomultiplier detectors were depth-adjusted.

\section{Morphological reconstructions.}

Morphologies of CA1 neurons were digitally 3D-reconstructed in a semi-automated manner with Imaris software (Bitplane, Inc.), using the AutoPath feature in the Filament Tracer module. Reconstructions were exported in Imaris format, converted into ASCII or SWT format using NLMorphologyViewer software (version 0.4.0.dev_x86.msi; NeuronLand, http://neuronland.org) and Matlab functions, and further analyzed using Matlab routines to align them and quantify length density ${ }^{58}$. Length-density maps of individual neurons were pooled and averaged. A contour plot of the resulting average map was used to determine the average 95\% contour level. Reconstructions will be made available at Neuromorpho.org.

\section{Immunohistochemistry.}

For immunostaining of CA1 slices, mice were first injected in the RSCg with retrograde tracer ( 50-100 nL), either red RetroBeads (undiluted; Lumafluor) or cholera toxin subunit B conjugated with Alexa647 (CTB647, $100 \mathrm{mg}$ in $20 \mu \mathrm{L}$ PBS; Invitrogen). After two or more days, to allow for retrograde transport, mice were transcardially perfused with ice-cold PBS (0.1M, pH 7.4) followed by $4 \%$ PFA (w/v in PBS). Brains were extracted and incubated in the same PFA solution overnight at $4{ }^{\circ} \mathrm{C}$. The brains were washed with PBS, and sliced 
(in PBS) into $100 \mu \mathrm{m}$ sections using a microtome (HV650 V, Microme). In some cases (SOM and CR), brains were cryoprotected in PBS containing sucrose (20\% then 30\%, w/v), and then frozen in OCT compound (Fisher), before being cut into $50 \mu \mathrm{m}$ section using a cryostat. Selected sections were transferred to a 24-well plate filled with PBS, and treated with 5\% normal donkey serum (NDS) and 0.3\% Triton X-100 in PBS for $60 \mathrm{~min}$. Sections were incubated overnight at $4^{\circ} \mathrm{C}$ in 5\% NDS and $0.3 \%$ Triton X-100 in PBS with antibodies to parvalbumin (anti-PV in mouse, 1:4000, Swant Cat\# 235, RRID:AB_10000343), calbindin (anti-CB in rabbit, 1:250, Swant Cat\# CB38, RRID:AB_2721225), calretinin (antiCR in goat, 1:4000, Swant Cat\# CG1, RRID:AB_10000342), reelin (anti-reelin in mouse, 1:1000, Millipore Cat\# MAB5364, RRID:AB_2179313), neuropeptide Y (anti-NPY in rabbit, 1:1000, ImmunoStar Cat\# 22940, RRID:AB_2307354), nitric oxide synthase (antinNOS in rabbit, 1:500, Millipore Cat\# AB5380, RRID:AB_91824), or somatostatin (antiSOM in rat, 1:1000, Millipore Cat\# MAB354, RRID:AB_2255365). After three washing steps in $1 \%$ NDS and $0.2 \%$ Triton X-100 in PBS, sections were incubated with a FITCconjugated secondary antibody (anti-rabbit or anti-goat in donkey, or anti-rat or anti-mouse in goat, as appropriate, 1:200; Molecular Probes Cat\# A-21206, RRID:AB_141708, Cat\# A-11055, RRID:AB_142672, Cat\# A-11029, RRID:AB_138404, Cat\# A-11006, RRID:AB_141373) in 5\% NDS and 0.3\% Triton X-100 in PBS for $2 \mathrm{~h}$. Cell nuclei were labeled with Hoechst (1:5.000, Sigma-Aldrich) in 1\% NDS and 0.2\% Triton X-100 in PBS for 15 minutes. Sections were rinsed twice, mounted with FluorSave and sealed with coverslip. A confocal microscope was used to capture z-stacks of images in regions of interest in CA1 containing the retrograde tracer and FITC signal (Olympus Fluoview FV10i, $10 \times$ objective lens, N/A $0.4,1024 \times 1024$ pixel, z-step size of 2 or $5 \mu \mathrm{m}$ ). Slices were tested for one antibody. Double-labeling of tracer and antibody staining was analyzed at the focal plane of retrogradely labeled neurons.

For amplification of hM4D(Gi)-mCherry signals, sections containing the RSCg and the injection site (i.e., CA1 or AV) were prepared using the same method as above, and were washed in $1 \% \mathrm{H}_{2} \mathrm{O}_{2}$ (v/v in methanol) and $5 \%$ goat serum (v/v in PBS) before being incubated overnight in chicken anti-mCherry antibody (1:16000 v/v in PBS, Abcam Cat\# ab205402, RRID:AB_2722769). Sections were washed in 1\% normal goat serum (v/v in PBS $)$ and incubated in biotinylated anti-chicken $\operatorname{IgG}(\mathrm{H}+\mathrm{L})$ made in goat $(1: 200 \mathrm{v} / \mathrm{v}$ in PBS, Vector Laboratories Cat\# BA-9010, RRID:AB_2336114) for 1 hour. The ABC kit (Vector Laboratories Cat\# PK-6100, RRID:AB_2336819) was used to amplify the immunosignal. Sections were further incubated in 3,3'-Diaminobenzidine (DAB; 1 tablet in $5 \mathrm{~mL} \mathrm{H}_{2} \mathrm{O}$, Sigma, D4293-50SET) for 7 mins, before being mounted on a slide and sealed with FluorSave and a \#1 coverslip.

The DAB staining was visualized using a macroscope (Olympus SZX16, equipped with a QImaging Retiga2000R camera). Images were acquired using Ephus software, without spatial binning.

\section{In vivo photostimulation and electrophysiology.}

The in vivo methods were recently described in detail ${ }^{59}$. Briefly, mice first underwent injection of $\mathrm{AAV}$ carrying $\mathrm{ChR} 2$ into either $\mathrm{CA} 1$ or $\mathrm{AV}$, using the same coordinates and 
methods as described above for ex vivo experiments. At least three weeks later, isofluraneanesthetized mice underwent placement of a head-post over the posterior fossa. A small ( $\varnothing$ $1 \mathrm{~mm}$ ) craniotomy was opened over the right RSC. Anesthesia was transitioned to ketaminexylazine (initial dose: ketamine 80-100 mg/kg, xylazine $5-15 \mathrm{mg} / \mathrm{kg}$, intraperitoneal). Mice were transferred to the recording rig, head-fixed, and thermally supported with heating pad (DC Temperature Control System, FHC). Anesthesia level was continually monitored based on whisking activity and reflexes (toe-pinch), and additional doses of ketamine/xylazine were given subcutaneously as needed to maintain a stable plane of anesthesia. ACSF was applied to keep exposed brain areas moist. An LED-coupled optical fiber (Thorlabs, FG400AEA $\varnothing 400 \mu \mathrm{m}$ core multimode fiber, $0.22 \mathrm{NA}, 6.1 \mathrm{~mW}$ output measured at the tip), mounted on a motorized micromanipulator, was positioned over the RSC, and photostimuli (10 ms-duration light pulses) were delivered every $2 \mathrm{~s}$. Trials were repeated 30 or more times. A two-shank linear array (coated with DiI, $0.5 \mathrm{~mm}$ separation between shanks, each with 16 channels with 1-2 M $\Omega$ impedance and 50- $\mu \mathrm{m}$ spacing; model A2 $\times 16-10 \mathrm{~mm}-50$ 500-177-A32, NeuroNexus, Ann Arbor, MI), mounted on a motorized 4-axis micromanipulator, was positioned at the cortical surface (coordinates of the anterior-most shank: $-1.6 \mathrm{~mm}$ caudal to bregma and $0.3-0.4 \mathrm{~mm}$ lateral to midline), and slowly inserted into the cortex to a depth of $1 \mathrm{~mm}$ from the pia. Signals were amplified using an RHD2132 digital electrophysiology amplifier board (Intan). We used a RHD2000 USB Interface Evaluation Board (Intan) streaming in the linear array data from the amplifier and piping the data stream in or out of the PC via USB cable. Recorded data, sampled at $30 \mathrm{KHz}$, were stored as raw signals from the amplifiers and filtered by a $60 \mathrm{~Hz}$ notch filter. A digital highpass filter ( $800 \mathrm{~Hz}$ cutoff, second-order Butterworth) was used to shrink the photovoltaic artifact to the first $3 \mathrm{~ms}$ post-stimulus window. We analyzed multi- rather than single-unit activity; single units could not be reliably isolated in within the barrages of activity evoked by optogenetic stimulation ${ }^{59}$. For this, a threshold detector was applied with threshold set to the 4 times the standard deviation to detect spike-like events. To mask the photovoltaic effect, spike counts of the $3 \mathrm{~ms}$ window from stimulus onset and offset were then replaced by null values (not-a-number, $\mathrm{NaN}$, in Matlab). These data from each shank were then combined and used to construct peristimulus time histograms, averaged across trials ${ }^{59}$. After each recording, mice were immediately sacrificed for post-hoc analysis of recording position and injection site. Mice with misplacement in any of these were excluded from analysis.

\section{Contextual fear conditioning and chemogenetic silencing.}

Thirty-eight mice, including 17 for vehicle (ACSF) and 21 for CNO infusion, were used for thalamic axonal silencing experiments. Twenty-nine mice, including 14 for vehicle and 15 for CNO infusion, were used for CA1 axonal silencing experiments. Mice previously injected with $\mathrm{hM} 4 \mathrm{D}(\mathrm{Gi})$-mCherry into either $\mathrm{CA} 1$ or thalamus were anesthetized with $1.2 \%$ tribromoethanol (v/v, Avertin) ${ }^{15}$ and craniotomy was performed bilaterally above the RSC. A guiding cannula (bilateral, short pedestal $(5 \mathrm{~mm}), 26$ gauge, 0.8 center-to-center distance, cut $0.75 \mathrm{~mm}$ below pedestal; PlasticOne) was slowly inserted into RSC and affixed to cranium using carboxylate cement (Durelon, 3M ESPE). A dummy cannula was inserted and a screw cap was attached to the pedestal to prevent clogging. Meloxicam $(1 \mathrm{mg} / \mathrm{kg})$ was injected subcutaneously for post-operative (48-72 h) reduction of pain and inflammation. Mice were single housed in cage and kept in a ventilated cabinet (Scantainer, Scanbur) for 1 
week before the day of experiment, a recovery time somewhat longer than in our previous studies (e.g. ref. ${ }^{60}$ ), and comparable to previous similar studies involving cannula implantation (e.g. ref. ${ }^{61}$ ).

For contextual fear conditioning, a behavioral paradigm that induces hippocampaldependent contextual fear memory in mice and modulates RSC activity ${ }^{60,62-66}$, mice were briefly exposed, for $3 \mathrm{~min}$, to context A: a $35 \times 20 \times 20 \mathrm{~cm}$ black box with a grid floor, an ethanol odor, and the room light on. This exposure was immediately followed by a foot shock ( $2 \mathrm{~s}, 0.7 \mathrm{~mA}$, constant current). This procedure results $40-60 \%$ freezing, allowing to observe modulatory effects of chemogenetic manipulations in either direction. Forty-eight hours later, mice were tested for fear memory retrieval to context, by placing them in context A for 3 mins. Freezing was scored every $10 \mathrm{~s}$ by different observers unaware of the experimental condition during context exposures, and expressed as a percentage of the total number of observations during which the mice were motionless with crouching posture. All behavioral experiments were performed between 10 am and $2 \mathrm{pm}$. Littermates were randomly assigned to the different treatment conditions (CNO or vehicle infusion, see below). All behavioral tests were performed with experimenters blind to genotypes and drug treatments.

Clozapine-N-oxide (CNO, Sigma; $1 \mathrm{mM}$ ) or vehicle was infused into RSC 30 mins before fear conditioning. Focal application of CNO blocks synaptic release from presynaptic terminals of axons expressing hM4D(Gi) ${ }^{67}$. The efficacy of this CNO concentration for silencing synaptic transmission at terminals was previously shown for subcortical ${ }^{68}$ and RSC circuits ${ }^{15}$ (and see below for additional characterizations). Mice were anesthetized with isoflurane, and the dummy cannula was replaced with an internal cannula connected to Hamilton microsyringes. An automatic microsyringe pump controller (Micro4-WPI) was used to infuse $\mathrm{CNO}$ or ACSF at a rate of $0.5 \mu \mathrm{L} / \mathrm{min}$ until a volume of $0.20 \mu \mathrm{L}$ per side was reached. After infusion, mice were placed back into the home cage for recovery before conditioning.

Caveats to the interpretation of these chemogenetic/behavioral experiments include the difficulty of entirely excluding the possibility that CNO diffused to other structures to influence $\mathrm{hM} 4 \mathrm{D}(\mathrm{Gi})$-expressing axons/somata outside the RSCg. This is particularly a potential concern for diffusion to the nearby subiculum, as a reconstructed CA1-RP $\rightarrow$ RSC axon was found to branch there ${ }^{10}$. ATN-TC $\rightarrow$ RSC axons, too, may have subicular branches (e.g. ${ }^{69}$; but see ref. ${ }^{16}$ ). However, in control experiments, when fluorescent muscimol was infused at the same rate and volume, the fluorescent signal was largely confined to the RSC, with little or no spread to hippocampus or other nearby structures (Supplementary Fig. S91p). These results are furthermore consistent with similar control experiments reported previously (see Fig. 2 of ref. ${ }^{61}$ ), and are broadly consistent with the original characterizations of the technique ${ }^{67}$. Nevertheless, caution is warranted in interpreting the chemogenetic/behavioral results given the difficulty of exactly determining the extent of $\mathrm{CNO}$ diffusion and its impact on synaptic transmission in vivo.

Post-hoc histological analysis was performed to confirm accurate targeting of the AAV to dorsal CA1 or AV (Supplementary Fig. S9j,k). After the test, mice were perfused and their 
brains were fixed and sectioned for mCherry amplification (see Immunohistochemistry section above). Sections were examined for cannula placement, injection site, and axonal projection pattern. Mice with a misplaced cannula, mis-targeted injection site, or weak mCherry expression were excluded from analysis $(n=6$ mice for CA1 silencing, $n=11$ mice for ATN silencing).

To control for the specificity of $\mathrm{CNO}$ and assess its efficacy in reducing synaptic transmission of $\mathrm{hM} 4 \mathrm{D}(\mathrm{Gi})$ expressing $\mathrm{CA} 1-\mathrm{RP} \rightarrow \mathrm{RSCg}$ and ATN-TC $\rightarrow \mathrm{RSCg}$ pathways, we injected AAV encoding hChR2 with or without AAV encoding hM4D(Gi) into CA1 of Gad2-Cre mice (Cre-dependent virus, 2:5 v/v ratio for hChR2 and hM4D(Gi)) or into thalamus of wild-type mice (Cre-independent virus, 1:5 v/v ratio). In RSCg slices, widefield photostimulation with an LED was used to activate ChR2-expressing axons while recording evoked synaptic currents from L5pyr neurons every $30 \mathrm{~s}$. After recording a stable baseline for 5 mins, $\mathrm{CNO}$ was added at an initial concentration of $0.1 \mu \mathrm{M}$, previously shown to be effective in hyperpolarizing $\mathrm{hM} 4 \mathrm{D}(\mathrm{Gi})$-expressing somata ${ }^{65}$ or silence synaptic transmission in other pathways ${ }^{15,67}$. After 7.5 mins, the $\mathrm{CNO}$ concentration was increased to $1 \mu \mathrm{M}^{67}$ (Supplementary Fig. S9a-i).

Drugs.

SR-95531 hydrobromide (Gabazine, \#1262), CGP55854 hydrochloride (\#1248), QX314 chloride (\#2313), NBQX (\#0373), ( $R$ )-CPP (\#0247) and TTX (\#1069) were purchased from R\&D Systems. 4-AP (275875-1G) and CNO (C0832-5MG) were purchased from SigmaAldrich. For ex vivo experiments, all drugs were dissolved in $\mathrm{H}_{2} \mathrm{O}$, except $\mathrm{NBQX}$ which was dissolved in DMSO. For in vivo experiments, $\mathrm{CNO}$ was dissolved in ACSF.

\section{Data collection and statistical analysis.}

Mice were randomly assigned to experimental groups. Data collection and analysis were not performed blind to the condition of experiment, except in behavioral studies (see above). Statistical analysis was performed using standard Matlab functions. Data distribution was assumed to be normal but this was not formally tested. Non-parametric tests such as signedrank (for paired data) or rank sum (unpaired data) tests were used to compare groups, unless otherwise noted, with significance defined as $\mathrm{p}<0.05$. All tests were 2-sided, allowing for the possibility of differences in either direction. No statistical methods were used to predetermine sample sizes, which are similar to those reported in previous publications ${ }^{15,70}$. The mean \pm s.e.m., unless otherwise noted, were used as statistical measures of central tendency and dispersion

\section{Supplementary Material}

Refer to Web version on PubMed Central for supplementary material.

\section{Acknowledgements:}

We thank N. Bernstein, A. Guedea, and D. Wokosin for a technical assistance and advice. We thank A. Apicella, J. Barrett, K. Guo, K. Harris, G. Maccaferri, and A. Tanimura for comments and suggestions. This research was supported by NIH grants NS061963 (G.S.), EB017695 (G.S.), and MH108837 (J.R.). 


\section{References (for main text only)}

1. Harris KD \& Shepherd GM The neocortical circuit: themes and variations. Nat Neurosci 18,170 181 (2015). [PubMed: 25622573]

2. Lin CS, Nicolelis MA, Schneider JS \& Chapin JK A major direct GABAergic pathway from zona incerta to neocortex. Science 248, 1553-1556 (1990). [PubMed: 2360049]

3. Chen J \& Kriegstein AR A GABAergic projection from the zona incerta to cortex promotes cortical neuron development. Science 350, 554-558 (2015). [PubMed: 26429884]

4. Saunders A, et al. A direct GABAergic output from the basal ganglia to frontal cortex. Nature 521, 85-89 (2015). [PubMed: 25739505]

5. Tomioka R, et al. Demonstration of long-range GABAergic connections distributed throughout the mouse neocortex. Eur J Neurosci 21, 1587-1600 (2005). [PubMed: 15845086]

6. He M, et al. Strategies and Tools for Combinatorial Targeting of GABAergic Neurons in Mouse Cerebral Cortex. Neuron 91, 1228-1243 (2016). [PubMed: 27618674]

7. Kimura F \& Baughman RW GABAergic transcallosal neurons in developing rat neocortex. Eur J Neurosci 9, 1137-1143 (1997). [PubMed: 9215696]

8. Rock C, Zurita H, Lebby S, Wilson CJ \& Apicella AJ Cortical Circuits of Callosal GABAergic Neurons. Cereb Cortex, 1-14 (2017). [PubMed: 28365777]

9. Miyashita T \& Rockland KS GABAergic projections from the hippocampus to the retrosplenial cortex in the rat. Eur J Neurosci 26, 1193-1204 (2007). [PubMed: 17767498]

10. Jinno $\mathrm{S}$, et al. Neuronal diversity in GABAergic long-range projections from the hippocampus. $\mathbf{J}$ Neurosci 27, 8790-8804 (2007). [PubMed: 17699661]

11. Vann SD, Aggleton JP \& Maguire EA What does the retrosplenial cortex do? Nat Rev Neurosci 10, 792-802 (2009). [PubMed: 19812579]

12. Gabriel M, Sparenborg SP \& Stolar N An executive function of the hippocampus: pathway selection for thalamic neuronal significance code in The Hippocampus (ed. Isaacson RL \& P. KH) (Springer, Boston, MA, 1986).

13. Corcoran KA, Yamawaki N, Leaderbrand K \& Radulovic J Role of retrosplenial cortex in processing stress-related context memories. Behav Neurosci (2018).

14. Sugar J, Witter MP, van Strien NM \& Cappaert NL The retrosplenial cortex: intrinsic connectivity and connections with the (para)hippocampal region in the rat. An interactive connectome. Front Neuroinform 5, 7 (2011). [PubMed: 21847380]

15. Yamawaki N, Corcoran KA, Guedea AL, Shepherd GMG \& Radulovic J Differential Contributions of Glutamatergic Hippocampal-->Retrosplenial Cortical Projections to the Formation and Persistence of Context Memories. Cereb Cortex (2018).

16. Odagiri S, Meguro R, Asano Y, Tani T \& Ichinohe N Single axon branching analysis in rat thalamocortical projection from the anteroventral thalamus to the granular retrosplenial cortex. Front Neuroanat 5, 63 (2011). [PubMed: 22013412]

17. van Groen T, Vogt BA \& Wyss JM Interconnections Between the Thalamus and Retrosplenial Cortex in the Rodent Brain in Neurobiology of Cingulate Cortex and Limbic Thalamus (ed. Vogt BA \& Gabriel M) (Birkhäuser, Boston, Massachusetts, 1993).

18. Price CJ, et al. Neurogliaform neurons form a novel inhibitory network in the hippocampal CA1 area. J Neurosci 25, 6775-6786 (2005). [PubMed: 16033887]

19. Overstreet-Wadiche L \& McBain CJ Neurogliaform cells in cortical circuits. Nat Rev Neurosci 16, 458-468 (2015). [PubMed: 26189693]

20. Price CJ, Scott R, Rusakov DA \& Capogna M GABA(B) receptor modulation of feedforward inhibition through hippocampal neurogliaform cells. J Neurosci 28, 6974-6982 (2008). [PubMed: 18596171]

21. Fuentealba P, et al. Expression of COUP-TFII nuclear receptor in restricted GABAergic neuronal populations in the adult rat hippocampus. J Neurosci 30, 1595-1609 (2010). [PubMed: 20130170]

22. Tasic B, et al. Adult mouse cortical cell taxonomy revealed by single cell transcriptomics. Nat Neurosci 19, 335-346 (2016). [PubMed: 26727548] 
23. Harris KD, et al. Classes and continua of hippocampal CA1 inhibitory neurons revealed by singlecell transcriptomics. PLoS Biol 16, e2006387 (2018). [PubMed: 29912866]

24. Gabriel M, Vogt BA, Kubota Y, Poremba A \& Kang E Training-stage related neuronal plasticity in limbic thalamus and cingulate cortex during learning: a possible key to mnemonic retrieval. Behav Brain Res 46, 175-185 (1991). [PubMed: 1664730]

25. Frank AC, et al. Hotspots of dendritic spine turnover facilitate clustered spine addition and learning and memory. Nature communications 9, 422 (2018).

26. Yamawaki N, Radulovic J \& Shepherd GM A Corticocortical Circuit Directly Links Retrosplenial Cortex to M2 in the Mouse. J Neurosci 36, 9365-9374 (2016). [PubMed: 27605612]

27. Wyss JM, Van Groen T \& Sripanidkulchai K Dendritic bundling in layer I of granular retrosplenial cortex: intracellular labeling and selectivity of innervation. J Comp Neurol 295, 33-42 (1990). [PubMed: 2341634]

28. Kurotani T, et al. Pyramidal neurons in the superficial layers of rat retrosplenial cortex exhibit a late-spiking firing property. Brain Struct Funct 218, 239-254 (2013). [PubMed: 22383041]

29. Ichinohe N, Fujiyama F, Kaneko T \& Rockland KS Honeycomb-like mosaic at the border of layers 1 and 2 in the cerebral cortex. J Neurosci 23, 1372-1382 (2003). [PubMed: 12598625]

30. Stepanyants A \& Chklovskii DB Neurogeometry and potential synaptic connectivity. Trends Neurosci 28, 387-394 (2005). [PubMed: 15935485]

31. Rees CL, Moradi K \& Ascoli GA Weighing the evidence in Peters' Rule: does neuronal morphology predict connectivity? Trends Neurosci 40, 63-71 (2017). [PubMed: 28041634]

32. Petreanu L, Mao T, Sternson SM \& Svoboda K The subcellular organization of neocortical excitatory connections. Nature 457, 1142-1145 (2009). [PubMed: 19151697]

33. Larkum M A cellular mechanism for cortical associations: an organizing principle for the cerebral cortex. Trends Neurosci 36, 141-151 (2013). [PubMed: 23273272]

34. Spruston N Pyramidal neurons: dendritic structure and synaptic integration. Nat Rev Neurosci 9 , 206-221 (2008). [PubMed: 18270515]

35. Anwar H, Li X, Bucher D \& Nadim F Functional roles of short-term synaptic plasticity with an emphasis on inhibition. Curr Opin Neurobiol 43, 71-78 (2017). [PubMed: 28122326]

36. Regehr WG Short-term presynaptic plasticity. Cold Spring Harbor perspectives in biology 4 , a005702 (2012). [PubMed: 22751149]

37. Aggleton JP \& Brown MW Episodic memory, amnesia, and the hippocampal-anterior thalamic axis. The Behavioral and brain sciences 22, 425-444; discussion 444-489 (1999). [PubMed: 11301518]

38. Wright NF, Vann SD, Erichsen JT, O’Mara SM \& Aggleton JP Segregation of parallel inputs to the anteromedial and anteroventral thalamic nuclei of the rat. J Comp Neurol 521, 2966-2986 (2013). [PubMed: 23504917]

39. Shibata $\mathrm{H}$ Efferent projections from the anterior thalamic nuclei to the cingulate cortex in the rat. $\mathrm{J}$ Comp Neurol 330, 533-542 (1993). [PubMed: 8320343]

40. Llinas R \& Jahnsen H Electrophysiology of mammalian thalamic neurones in vitro. Nature 297 , 406-408 (1982). [PubMed: 7078650]

41. Jiang X, Wang G, Lee AJ, Stornetta RL \& Zhu JJ The organization of two new cortical interneuronal circuits. Nat Neurosci 16, 210-218 (2013). [PubMed: 23313910]

42. Llinas R \& Ribary U Coherent $40-\mathrm{Hz}$ oscillation characterizes dream state in humans. Proc Natl Acad Sci U S A 90, 2078-2081 (1993). [PubMed: 8446632]

43. Takahashi H \& Magee JC Pathway interactions and synaptic plasticity in the dendritic tuft regions of CA1 pyramidal neurons. Neuron 62, 102-111 (2009). [PubMed: 19376070]

44. Higley MJ Localized GABAergic inhibition of dendritic Ca(2+) signalling. Nat Rev Neurosci 15, 567-572 (2014). [PubMed: 25116141]

45. Murayama M, et al. Dendritic encoding of sensory stimuli controlled by deep cortical interneurons. Nature 457, 1137-1141 (2009). [PubMed: 19151696]

46. Egger R, et al. Robustness of sensory-evoked excitation is increased by inhibitory inputs to distal apical tuft dendrites. Proc Natl Acad Sci U S A 112, 14072-14077 (2015). [PubMed: 26512104] 
47. Ranganath C \& Ritchey M Two cortical systems for memory-guided behaviour. Nat Rev Neurosci 13, 713-726 (2012). [PubMed: 22992647]

48. Viney TJ, et al. Shared rhythmic subcortical GABAergic input to the entorhinal cortex and presubiculum. eLife 7 (2018).

49. Llinas RR, Ribary U, Jeanmonod D, Kronberg E \& Mitra PP Thalamocortical dysrhythmia: A neurological and neuropsychiatric syndrome characterized by magnetoencephalography. Proc Natl Acad Sci U S A 96, 15222-15227 (1999). [PubMed: 10611366]

\section{Methods-only References}

50. Taniguchi $\mathrm{H}$, et al. A resource of Cre driver lines for genetic targeting of GABAergic neurons in cerebral cortex. Neuron 71, 995-1013 (2011). [PubMed: 21943598]

51. Peron SP, Freeman J, Iyer V, Guo C \& Svoboda K A Cellular Resolution Map of Barrel Cortex Activity during Tactile Behavior. Neuron 86, 783-799 (2015). [PubMed: 25913859]

52. Gerfen CR, Paletzki R \& Heintz N GENSAT BAC cre-recombinase driver lines to study the functional organization of cerebral cortical and basal ganglia circuits. Neuron 80, 1368-1383 (2013). [PubMed: 24360541]

53. Tervo DG, et al. A designer AAV variant permits efficient retrograde access to projection neurons. Neuron 92, 372-382 (2016). [PubMed: 27720486]

54. Suter BA, et al. Ephus: multipurpose data acquisition software for neuroscience experiments. Frontiers in Neuroscience Methods 4, 1-12 (2010).

55. Yamawaki N, Suter BA, Wickersham IR \& Shepherd GMG Combining optogenetics and electrophysiology to analyze projection neuron circuits. Cold Spring Harb Protoc 2016, pdb prot090084 (2016).

56. Suter BA \& Shepherd GMG Reciprocal interareal connections to corticospinal neurons in mouse $\mathrm{m} 1$ and s2. J Neurosci 35, 2959-2974 (2015). [PubMed: 25698734]

57. Jackman SL, Beneduce BM, Drew IR \& Regehr WG Achieving high-frequency optical control of synaptic transmission. J Neurosci 34, 7704-7714 (2014). [PubMed: 24872574]

58. Yamawaki N, Borges K, Suter BA, Harris KD \& Shepherd GMG A genuine layer 4 in motor cortex with prototypical synaptic circuit connectivity. eLife 3, e05422 (2014). [PubMed: 25525751]

59. Li X, Yamawaki N, Barrett JM, Kording KP \& Shepherd GMG Scaling of optogenetically evoked signaling in a higher-order corticocortical pathway in the anesthetized mouse. Front Syst Neurosci 12, 16 (2018). [PubMed: 29867381]

60. Corcoran KA, et al. NMDA receptors in retrosplenial cortex are necessary for retrieval of recent and remote context fear memory. J Neurosci 31, 11655-11659 (2011). [PubMed: 21832195]

61. Nelson AJ, Powell AL, Holmes JD, Vann SD \& Aggleton JP What does spatial alternation tell us about retrosplenial cortex function? Frontiers in behavioral neuroscience 9, 126 (2015). [PubMed: 26042009]

62. Cowansage KK, et al. Direct reactivation of a coherent neocortical memory of context. Neuron 84 , 432-441 (2014). [PubMed: 25308330]

63. Tanaka KZ, et al. Cortical representations are reinstated by the hippocampus during memory retrieval. Neuron 84, 347-354 (2014). [PubMed: 25308331]

64. Kwapis JL, Jarome TJ, Lee JL \& Helmstetter FJ The retrosplenial cortex is involved in the formation of memory for context and trace fear conditioning. Neurobiol Learn Mem 123, 110-116 (2015). [PubMed: 26079095]

65. Jovasevic V, et al. GABAergic mechanisms regulated by miR-33 encode state-dependent fear. Nat Neurosci 18, 1265-1271 (2015). [PubMed: 26280760]

66. Keene CS \& Bucci DJ Contributions of the retrosplenial and posterior parietal cortices to cuespecific and contextual fear conditioning. Behav Neurosci 122, 89-97 (2008). [PubMed: 18298252]

67. Stachniak TJ, Ghosh A \& Sternson SM Chemogenetic synaptic silencing of neural circuits localizes a hypothalamus-->midbrain pathway for feeding behavior. Neuron 82, 797-808 (2014). [PubMed: 24768300] 
68. Mahler SV, et al. Designer receptors show role for ventral pallidum input to ventral tegmental area in cocaine seeking. Nat Neurosci 17, 577-585 (2014). [PubMed: 24584054]

69. Van Groen T \& Wyss JM Projections from the anterodorsal and anteroventral nucleus of the thalamus to the limbic cortex in the rat. J Comp Neurol 358, 584-604 (1995). [PubMed: 7593752]

70. Yamawaki N \& Shepherd GMG Synaptic circuit organization of motor corticothalamic neurons. J Neurosci 35, 2293-2307 (2015). [PubMed: 25653383]

71. Ohashi K, et al. Neuron-derived neurotrophic factor functions as a novel modulator that enhances endothelial cell function and revascularization processes. J Biol Chem 289, 14132-14144 (2014). [PubMed: 24706764] 

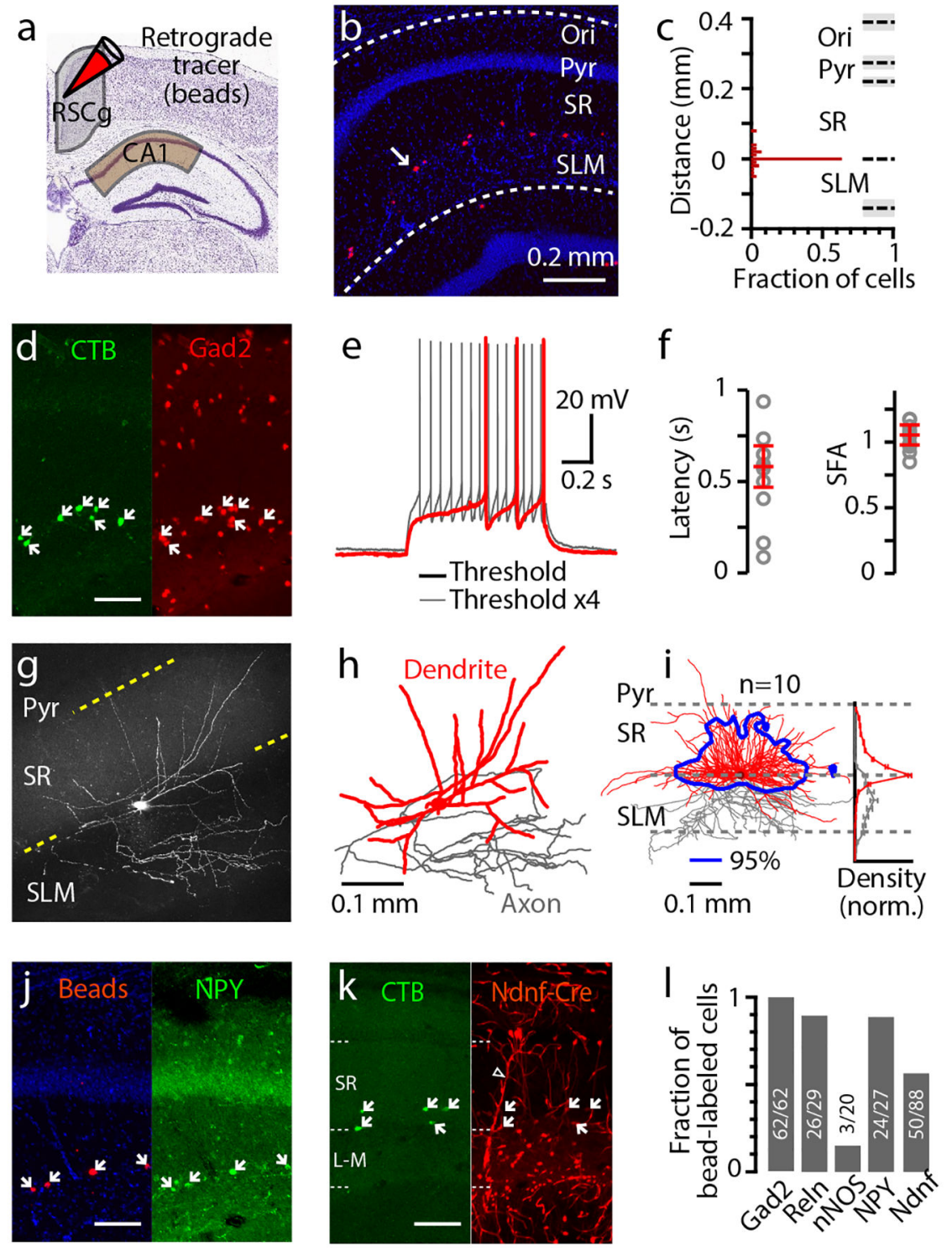

Fig. 1. CA1 retrosplenial-projecting (CA1-RP) neurons are a distinct class of GABAergic neurons.

a, Schematic of tracer injection into the granular retrosplenial cortex (RSCg) to label RSCgprojecting neurons in dorsal CA1.

b, Retrogradely labeled neurons (red) in CA1 after tracer injection in RSCg (one labeled neuron indicated with arrow). The slice was also labeled with DAPI (blue) to identify layers; Ori: oriens, Pyr: pyramidale, SR: radiatum, SLM: lacnosum-moleculare. The image is one of 7 hippocampi from 2 mice used for quantification in $\mathbf{c}$.

c, Histogram of the laminar distribution of CA1-RP neurons ( 2 mice, 3 and 4 slices each) relative to the stratum radiatum/lacunosum-moleculare (SR-SLM) border. Bar: $10 \mu \mathrm{m}$ bin. d, Example image of CA1 showing retrogradely labeled RSCg-projecting neurons (with $\mathrm{CTB}$, green) and genetically labeled Gad2-positive neurons (with mCherry, red). White arrows indicate neurons co-labeled with CTB. Scale bar: $0.1 \mathrm{~mm}$. The image is one of 6 hippocampi from 2 mice used for quantification in $\mathbf{l}$. 
e, Typical spiking pattern exhibited by CA1-RP neurons at rheobase (red) and above rheobase (gray).

f, Left: Plot of latency to first spike, from start of current injection step, at rheobase ( $\mathrm{n}=10$ neurons). Right: Plot of spike frequency adaptation ratio, measured for the first trace with $\geq 5$ spikes as the ratio of the $4^{\text {th }} / 1^{\text {st }}$ interspike interval (same group of 10 neurons). Error bars: mean \pm s.e.m.

g, Example 2-photon image (maximum intensity projection) of CA1-RP neurons (slice thickness: $300 \mu \mathrm{m})$. See next panel for scale bar. In total, $\mathrm{n}=10$ neurons were imaged and reconstructed.

h, Reconstructed dendritic (red) and local axonal (gray) morphology of the neuron in previous panel.

i, Reconstructed dendrites from ten CA1-RP neurons were overlaid and aligned. Blue contour: 95\% confidence interval of dendritic territory. Right: laminar profiles of dendritic (red) and axonal (gray) length density (normalized to peak).

j, Example image of CA1 showing retrogradely labeled RSCg-projecting neurons (with beads, red), and immuno-labeled NPY-positive neurons (with FITC, green). White arrows indicate neurons co-labeled with beads. Scale bar: $0.1 \mathrm{~mm}$. The image is one of 3 hippocampi from 2 mice used for quantification in $\mathbf{l}$.

$\mathbf{k}$, Example image of CA1 showing retrogradely labeled RSCg-projecting neurons (CTB, green), and genetically labeled Ndnf-positive neurons (tdTomato, red). White arrows indicate neurons co-labeled with CTB. Laminar boundaries are shown with dotted lines. Hollow arrowhead indicates endothelial-like structures, which also express $\mathrm{Ndnf}^{71}$. Scale bar: $0.1 \mathrm{~mm}$. The image is one of 11 hippocampi from 2 mice used for quantification in $\mathbf{I}$. 1, Fractions of retrogradely labeled neurons co-labeled with different markers (total number of co-labeled neurons/total number of retrogradely labeled neurons). 


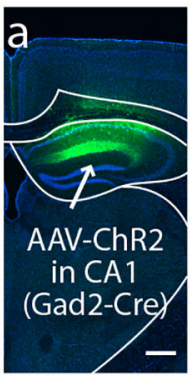

e
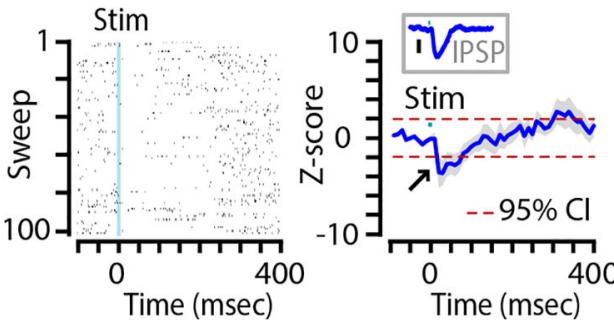

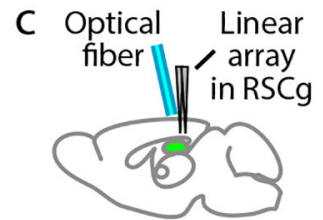

AAV-ChR2 in

CA1 (Gad2-Cre)
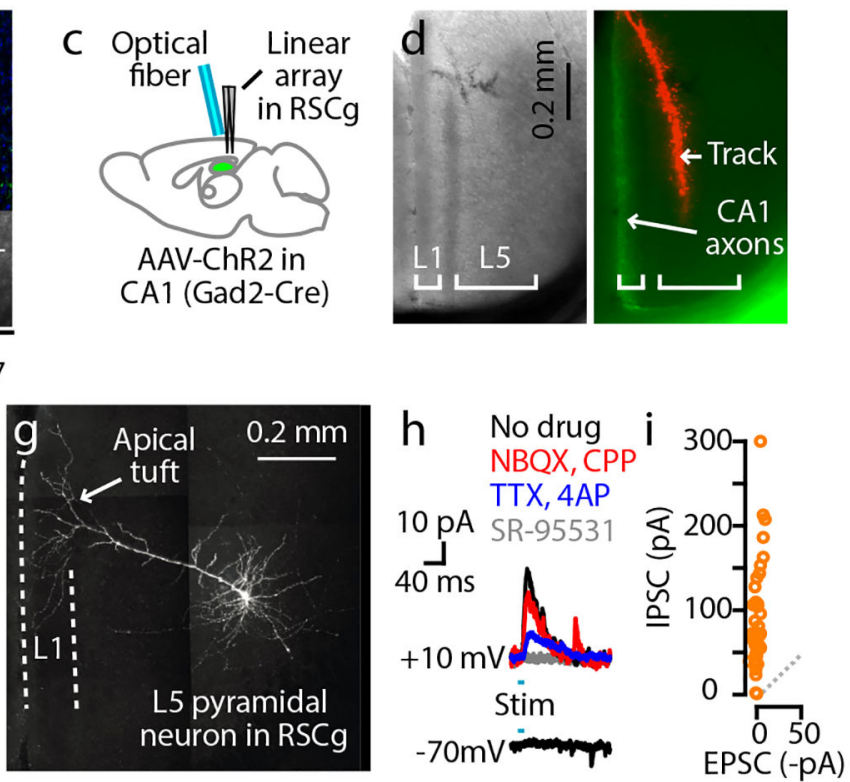
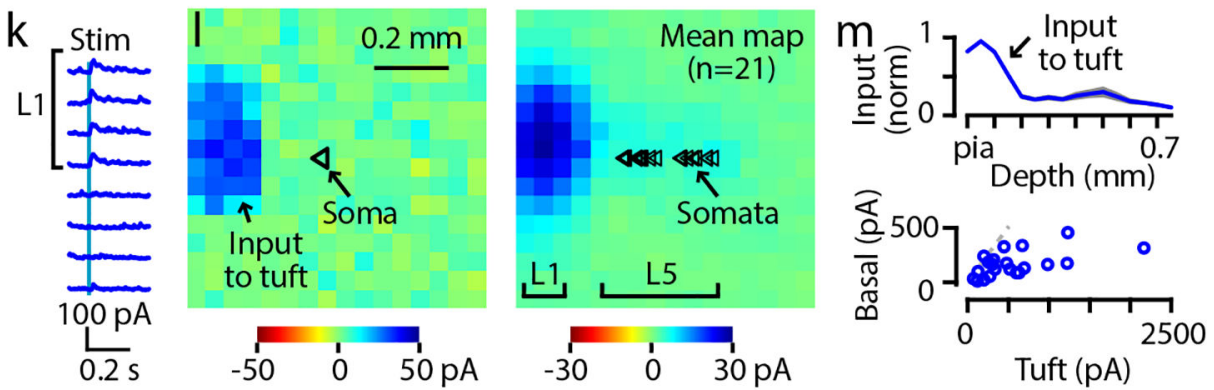

Tuft (pA)

Fig. 2. CA1-RP neurons form inhibitory synapses onto RSCg-L5pyr neurons at L1 apical tuft dendrites.

a, Coronal section showing site of Cre-dependent AAV5-Ef1a-DIO-hChR2-EYFP injection in CA1 of a Gad2-Cre mouse. Scale bar: $0.5 \mathrm{~mm}$.

b, Bright-field (bottom) and confocal (top) images of RSCg in a coronal section from the same CA1-injected mouse, showing L1, L5, and hChR2-expressing CA1 axons (green). Similar observation was made in all experiments concerning $\mathrm{CA} 1 \rightarrow \mathrm{RSCg}$ projection conducted in this study.

c, Schematic of the in vivo recording arrangement.

d, Bright-field (left) and epifluorescence (right) images of the RSCg, showing CA1-RP axons in L1 and a DiI-marked electrode track in L5. Similar observation in 7 other mice.

e, Example of peristimulus multi-unit activity recorded on one of the probe's channels across multiple sweeps.

f, Plot of the averaged z-score computed from PSTHs (blue: mean; gray: \pm s.e.m.; $\mathrm{n}=8$ mice; red dotted lines: 95\% confidence interval; 10-ms bins). Inset: Mean inhibitory postsynaptic potential (IPSP) waveform (blue, \pm s.e.m., gray), for comparison. IPSPs, recorded in drug-free ACSF from L5pyr neurons $(n=7)$ in $\mathrm{RSCg}$ slices ex vivo, were evoked by photostimulating CA1-RP axons in L1 with a focused laser (1 ms pulse duration). Traces were baseline-subtracted before averaging (mean resting membrane potential: -62.2 $\pm 1.6 \mathrm{mV}$ ). Bar: $0.1 \mathrm{mV}$. 
g, 2-photon image (maximum intensity projection) of a recorded RSCg-L5pyr neuron (coronal slice thickness: $250 \mu \mathrm{m}$ ), with prominent apical dendritic tuft in L1 (arrow). Similar observation in 4 other neurons.

h, Example traces showing post-synaptic currents evoked by photostimulation of CA1-RP axons at different command potentials (-70 $\mathrm{mV}$ for EPSC and $+10 \mathrm{mV}$ for IPSC) and in different pharmacological conditions. Group data is in $\mathbf{i}$.

i, Pairwise comparison of EPSC and IPSC recorded from multiple RSCg-L5pyr neurons after photostimulation of CA1-RP axons in drug-free condition (mean \pm s.e.m.; $0.1 \pm 0.5 \mathrm{pA}$ vs $85.2 \pm 9.4 \mathrm{pA} ; \mathrm{n}=42$ neurons, $\mathrm{p}=2.0 \mathrm{e}-08$, signed-rank test).

j, Schematic of the sCRACM method. Whole-cell recordings were made from RSCg-L5pyr neurons in slices containing ChR2-expressing CA1-RP axons, while a focused laser beam was scanned sequentially across the sites in the stimulation grid $(16 \times 16,50 \mu \mathrm{m}$ spacing $)$ to generate an input map (see Methods).

$\mathbf{k}$, Example peristimulus current traces from the map shown in the next panel (row 7 , columns 1-8).

l, Example (left) and mean (right; $\mathrm{n}=21$ neurons) sCRACM maps of CA1-RP inhibitory input to RSCg-L5pyr dendrites. Triangles: soma positions of the recorded neurons.

m, Top: Laminar profile of CA1-RP inhibitory input (normalized to peak, mean \pm s.e.m.). Bottom: Pairwise comparison of input to dendrites in L1 (left-most 3 columns) vs basal/ perisomatic region (3 columns around the soma). 

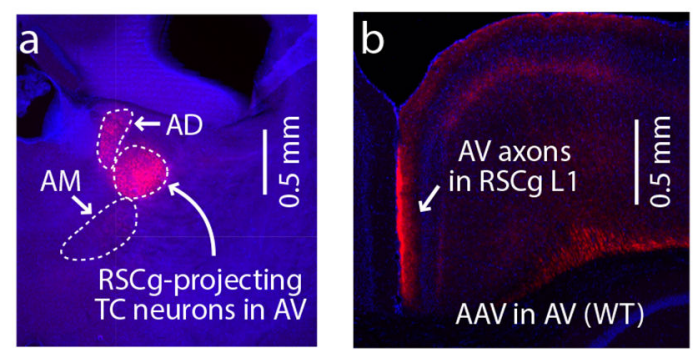

e

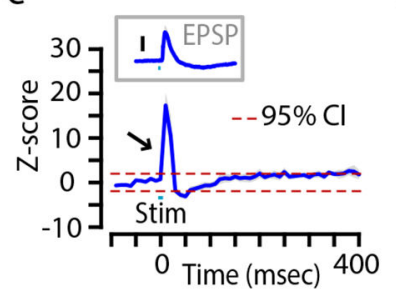

$f$
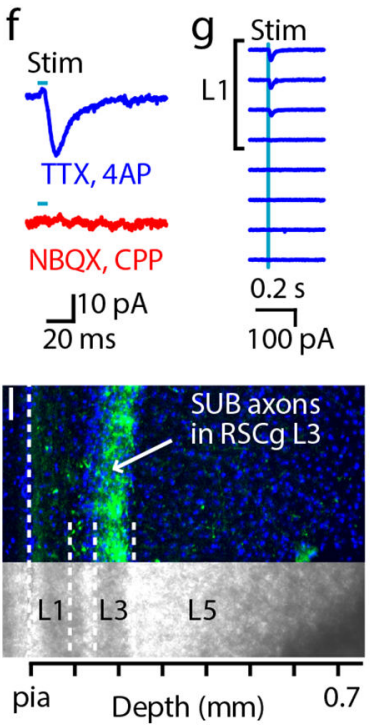
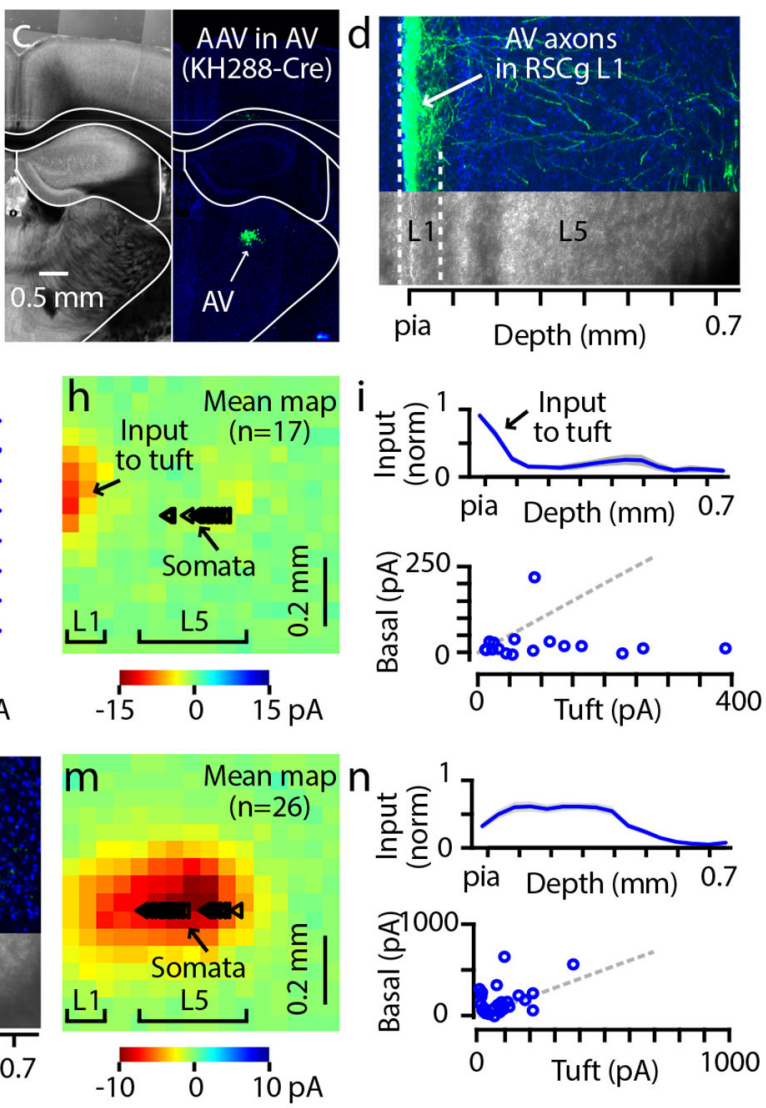

Fig. 3. ATN-TC axons make excitatory synapses onto RSCg-L5pyr neurons at L1 apical tuft dendrites.

a, Coronal section showing labeling in $\mathrm{AV}$ and the anterodorsal (AD) nucleus after retrograde CTB647 injection into RSCg. AM: anteromedial nucleus. Repeated at least on 3 different mice.

b, Axonal projection pattern in RSC resulting from AAV1-CamKIIa-hChR2-mCherry injection targeted to AV (coronal section). Similar observation was made in all experiments concerning $\mathrm{ATN} \rightarrow \mathrm{RSCg}$ projection conducted in this study.

c, Labeling in AV (Grp_KH288-Cre mouse) at the site of injection of AAV5-Ef1a-DIOhChR2-EYFP.

d, Axonal projection to RSCg resulting from the injection shown in the previous panel. c-d was repeated at least on 6 different mice.

e, Plot showing averaged z-score computed from PSTH (blue). Dotted lines (red) indicate 95\% confidence interval. Bin: $10 \mathrm{~ms}$. Inset: Mean excitatory postsynaptic potential (EPSP) waveform (blue, \pm s.e.m., gray), for comparison, recorded in drug-free ACSF from L5pyr neurons $(\mathrm{n}=7)$ in RSCg slices ex vivo, while focally photostimulating ATN-TC axons in L1 with a focused laser. Traces were baseline-subtracted before averaging (mean resting membrane potential: $-64.3 \pm 2.8 \mathrm{mV}$ ). Bar: $0.5 \mathrm{mV}$.

f, Example EPSC recorded in a RSCg-L5pyr neuron after photostimulation of ATN-TC axons in different pharmacological conditions ( $\mathrm{n}=5$ neurons).

g, Example peristimulus current traces extracted from one neuron's sCRACM map. 
h, Mean sCRACM map of ATN-TC excitatory synaptic input to RSCg-L5pyr dendrites ( $\mathrm{n}=$ 17 neurons). Triangles: soma positions of the recorded neurons.

i, Top: Laminar profile of ATN-TC excitatory input (normalized to peak, mean \pm s.e.m.). Bottom: Pairwise comparison of input to dendrites in L1 (left-most 3 columns) vs basal/ perisomatic region (3 columns around the soma).

j, Example traces of photo-evoked PSPs recorded from two different L5pyr neurons, before (black) and after (red) application of SR-95531 to block CA1-RP inputs. In one neuron (top), SR-95531 changed the photo-evoked response from net inhibitory to excitatory; in another neuron (bottom), it enhanced the size of EPSP.

$\mathbf{k}$, Comparison of PSP amplitude (mean of $30 \mathrm{~ms}$ from stim onset) before and after SR-95531. Gray lines are for individual recordings, and black indicates the group mean $(\mathrm{n}=$ 11 neurons).

l, Axonal projection pattern in RSCg resulting from injection of AAV1-CamKIIa-hChR2-

mCherry into dorsal subiculum. Similar observation was made in all experiments concerning subiculum $\rightarrow$ RSCg projection conducted in this study.

$\mathbf{m}$, Mean sCRACM map of subicular excitatory synaptic input to RSCg-L5pyr dendrites ( $\mathrm{n}=$ 26 neurons). Triangles: soma positions of the recorded neurons.

n, Same as panel i, but for subicular inputs. 


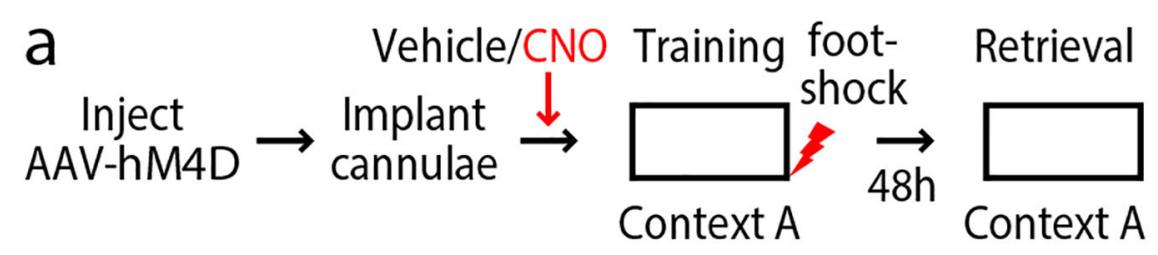

$\mathrm{b}$
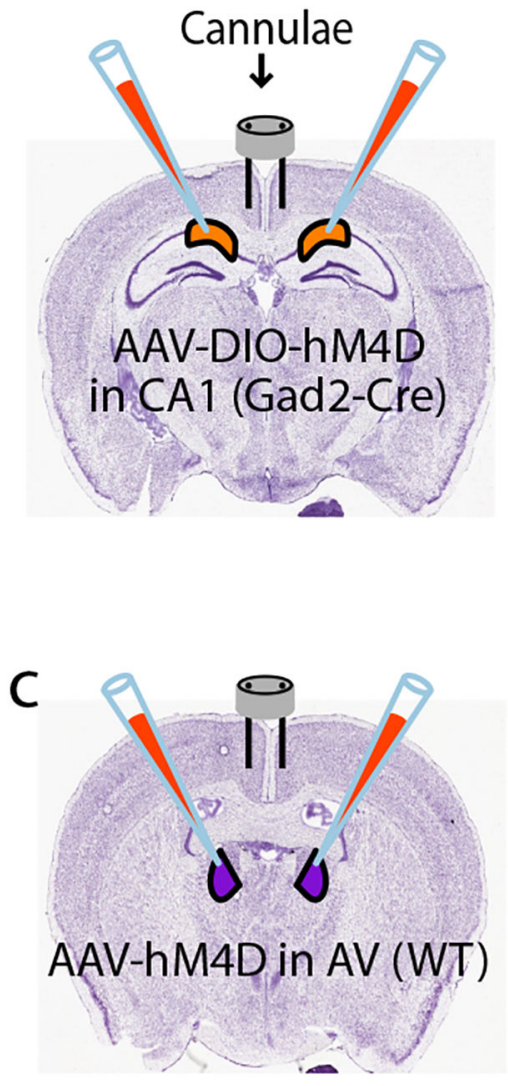

- Vehicle $\mathbf{C N O}$

$p=0.029$
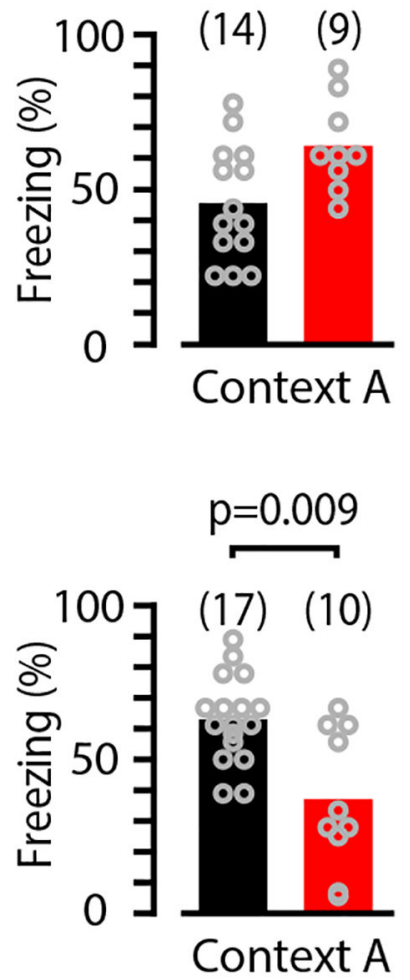

Fig. 4. Opposing actions of CA1-RP inhibition and ATN-TC excitation in contextual fear learning.

a, Schematic of the experimental paradigm.

b, Left: Schematic illustrating bilateral injection of AAV8-hSyn-DIO-hM4D(Gi)-mCherry into CA1 of Gad2-cre mice and placement of cannulae in RSC for CNO/ACSF infusion during CFC. Right: Freezing score (\%) during exposure of mice $(n=14$ and 9 mice for vehicle and CNO group, respectively; vehicle group consisted of 8 female and 6 male mice; $\mathrm{CNO}$ group consisted of 5 female and 4 male mice; $\mathrm{p}=0.03$, rank-sum test) to conditioned context during retrieval testing.

c, Left: Schematic illustrating bilateral injection of AAV8-hSyn-HA-hM4D(Gi)-mCherry into AV of wild-type mice. Right: same as B, but for assessment of ATN $\rightarrow$ RSC connections in CFC ( $\mathrm{n}=17$ and 10 mice for vehicle and CNO group, respectively; vehicle group consisted of 7 female and 3 male mice; CNO group consisted of 7 female and 3 male mice; $\mathrm{p}=0.009$, rank-sum test). 


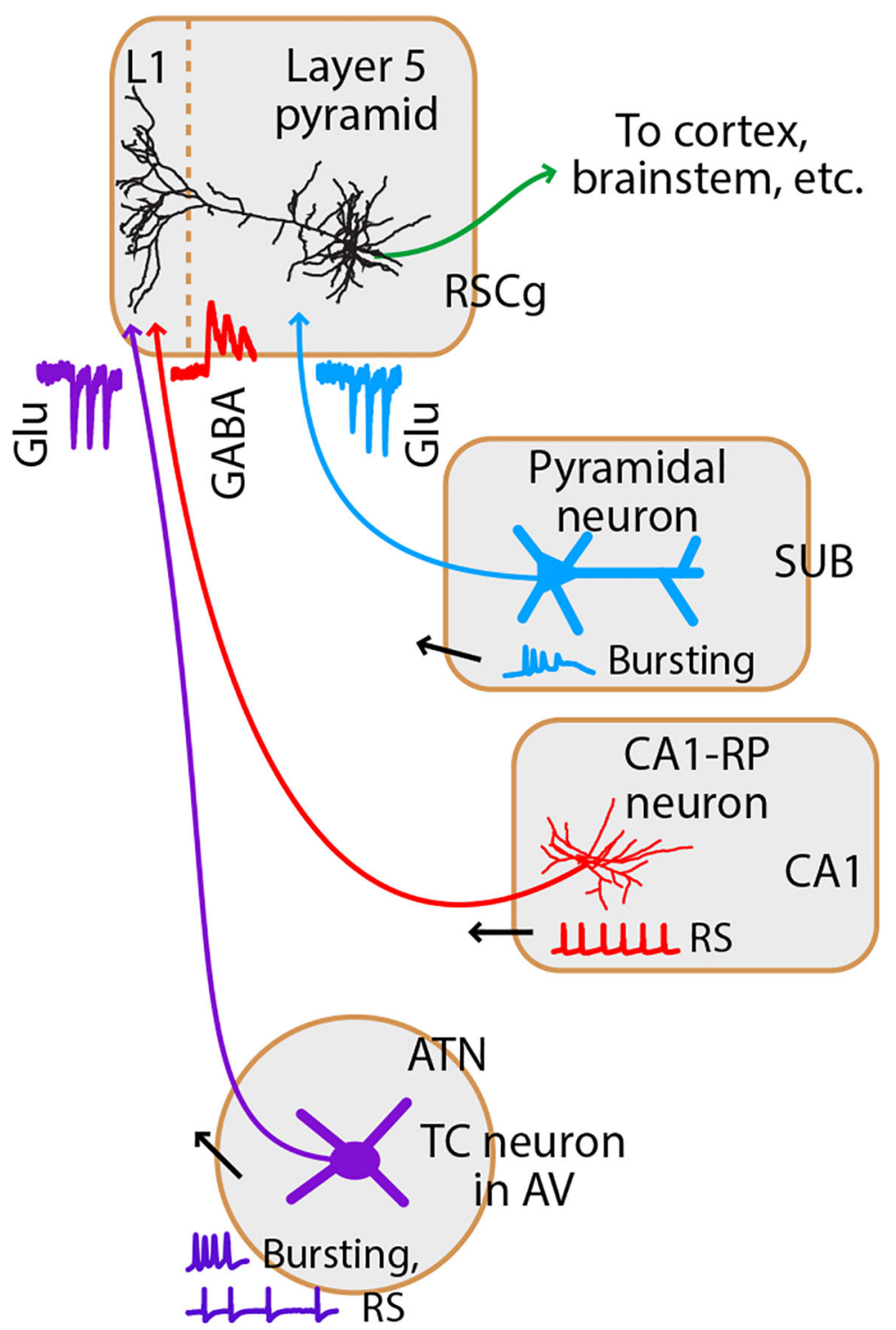

Fig. 5. Cellular and subcellular organization of long-range input circuits of the RSCg. Inhibitory axons from CA1-RP neurons and excitatory axons from ATN thalamocortical neurons, particularly in the AV nucleus, converge in L1 of RSCg and innervate the distal, apical-tuft dendrites of L5pyr neurons, with opposing neurotransmitter actions. Excitatory axons from dorsal subiculum innervate more proximal dendrites in deeper layers. Activitydependent synaptic properties (traces near the synaptic connections) and somatic firing patterns (traces near presynaptic somata) are distinct for each source of afferent input. RS: regular-spiking. 